ARTICLE

https://doi.org/10.1038/s41467-019-10835-4

\title{
Dietary fatty acids promote lipid droplet diversity through seipin enrichment in an ER subdomain
}

\author{
Zhe Cao (i) ${ }^{1}$, Yan Hao ${ }^{1}$, Chun Wing Fung (10 1, Yiu Yiu Lee ${ }^{1}$, Pengfei Wang ${ }^{2}$, Xuesong Li (D) ${ }^{3}$, Kang Xie ${ }^{4}$,

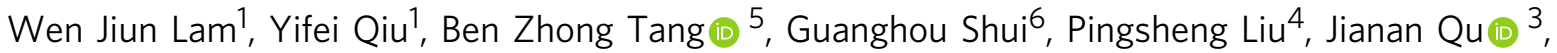 \\ Byung-Ho Kang (D) ${ }^{2} \&$ Ho Yi Mak (D) ${ }^{1}$
}

Exogenous metabolites from microbial and dietary origins have profound effects on host metabolism. Here, we report that a sub-population of lipid droplets (LDs), which are conserved organelles for fat storage, is defined by metabolite-modulated targeting of the $C$. elegans seipin ortholog, SEIP-1. Loss of SEIP-1 function reduces the size of a subset of LDs while over-expression of SEIP-1 has the opposite effect. Ultrastructural analysis reveals SEIP-1 enrichment in an endoplasmic reticulum (ER) subdomain, which co-purifies with LDs. Analyses of $C$. elegans and bacterial genetic mutants indicate a requirement of polyunsaturated fatty acids (PUFAs) and microbial cyclopropane fatty acids (CFAs) for SEIP-1 enrichment, as confirmed by dietary supplementation experiments. In mammalian cells, heterologously expressed SEIP-1 engages nascent lipid droplets and promotes their subsequent expansion in a conserved manner. Our results suggest that microbial and polyunsaturated fatty acids serve unexpected roles in regulating cellular fat storage by promoting LD diversity.

\footnotetext{
${ }^{1}$ Division of Life Science, The Hong Kong University of Science and Technology, Hong Kong SAR, China. ${ }^{2}$ School of Life Science, The Chinese University of Hong Kong, Hong Kong SAR, China. ${ }^{3}$ Biophotonics Research Laboratory, Department of Electronic and Computer Engineering, The Hong Kong University of Science and Technology, Hong Kong SAR, China. ${ }^{4}$ Institute of Biophysics, Chinese Academy of Sciences, Beijing, China. ${ }^{5}$ Department of Chemistry, The Hong Kong University of Science and Technology, Hong Kong SAR, China. ${ }^{6}$ Institute of Genetics and Developmental Biology, Chinese Academy of Sciences, Beijing, China. Correspondence and requests for materials should be addressed to H.Y.M. (email: hym@ust.hk)
} 
ipid droplets (LDs) are the primary organelles for fat storage in all eukaryotic cells ${ }^{1-3}$. They accommodate excess energy in the forms of triglycerides (TAG) and cholesterol esters (CE) within a phospholipid monolayer that is enriched in phosphatidylcholine or phosphatidylethanolamine $e^{4,5}$. Extensive proteomic analyses revealed a core set of proteins that reside on the surface of LDs, some of which regulate fat deposition and mobilization $^{6}$. Although LDs play a central role for intracellular fat storage, they are known to accommodate additional cargos in a developmental stage- and tissue-specific manner ${ }^{7-9}$. The diversification of LDs is further hinted by the preferential association of metabolic enzymes to subsets of $\mathrm{LDs}^{10,11}$. However, the molecular mechanisms that underlie LD diversity are not fully understood.

In the energy-replete state, LDs are closely associated with the endoplasmic reticulum (ER) ${ }^{12,13}$, which can be facilitated by protein-protein interactions or through stable or transient membrane connections ${ }^{11,14-17}$. The physical coupling of the two organelles is required for $\mathrm{LD}$ expansion, in part because TAG precursors are made in the ER before they are converted into TAG by DGAT2 at the ER-LD interface ${ }^{11,14,18,19}$. Additional evidence for a fundamental role of the ER in cellular fat storage comes from the discovery that Atlastins regulate LD size $\mathrm{e}^{20}$. Atlastins are conserved proteins essential for the generation and maintenance of the tubular ER network. Depletion of Atlastins reduces $\mathrm{LD}$ size $\mathrm{e}^{20}$, possibly due to a loss of tubular ER subdomains that are dedicated for ER-LD coupling. However, how such subdomains are maintained and segregated from the rest of the network is unknown.

The seipin protein family plays a conserved role in lipid homeostasis and maintenance of lipid droplet morphology. Mutations in seipin/BSCL2 cause a severe form of congenital generalized lipodystrophy in humans ${ }^{21,22}$. Pleiotropic metabolic defects such as insulin resistance, hypertriglyceridemia, and hepatic steatosis are thought to stem from an almost complete lack of adipose tissues. Accordingly, seipin has been shown to act cell-autonomously to promote adipocyte differentiation ${ }^{23-25}$. Biochemical and structural studies suggest that seipin assembles into homo-oligomers ${ }^{26-28}$. Intriguingly, seipin orthologs were found at ER-LD junctions where it may be responsible for the proper partitioning of lipids and proteins between the ER and LDs ${ }^{22,29-32}$. In addition, loss of seipin function in yeast and Drosophila alters the profile of acyl chains in phospholipids ${ }^{30,33}$, which has been proposed to cause gross disturbance to LD morphology, resulting in 'supersized' LDs or clusters of abnormally small and misshapen $\mathrm{LDs}^{30,32}$. More recently, a role for seipin in the maturation of nascent LDs has been reported ${ }^{34}$. Therefore, the precise localization of seipin appears to be critical for its function.

The phospholipid composition is a major determinant of the structure and function of eukaryotic membrane-bound organelles ${ }^{35}$. The synthesis of phospholipids is in turn dependent on the availability of exogenous and endogenous precursors of head groups and fatty acyl chains in the form of fatty acids ${ }^{36}$. In humans, dietary polyunsaturated fatty acids are readily incorporated into membrane phospholipids. They modulate membrane fluidity and serve as precursors for key immunomodulatory molecules. In addition to the diet, the gut microbiome is another potential source of exogenous fatty acids. For example, bacterial cyclopropane fatty acids are found to be incorporated into phospholipids and triglycerides in human adipose tissue ${ }^{37}$. However, the significance of bacterial fatty acids in eukaryotic membranes is so far obscure.

In this paper, we report that human seipin and its C. elegans ortholog SEIP-1 are targeted to a tubular ER subdomain. SEIP-1 is enriched in ER tubules that tightly associate with a subset of
LDs. Proper targeting of SEIP-1 is critically dependent on specific polyunsaturated fatty acids (PUFAs) and bacterial cyclopropane fatty acids. Therefore, LDs heterogeneity may originate from fatty acid-driven targeting of distinct protein ensembles.

\section{Results}

SEIP-1 regulates LD size. A single seipin ortholog, SEIP-1, was identified in the $C$. elegans proteome by BLAST. Membrane topology predictions suggested that, similar to human seipin, SEIP-1 has two transmembrane helices flanking a conserved central loop region, which may reside in the ER lumen (Supplementary Fig. 1a) ${ }^{38,39}$. To elucidate the function of SEIP-1 in C. elegans, we examined seip-1(tm4221) mutant animals, which harbored a deletion that removed exon 4 and part of exon 3 of the R01B10.6 open reading frame (Fig. 1a). We could not detect full-length SEIP-1 by western blotting in these animals (Fig. 1d). Therefore, $t m 4221$ is likely a molecular null allele. We focused our phenotypic analysis on the intestine, which is the major site of fat storage in C. elegans ${ }^{2}$. Using label-free stimulated Raman scattering (SRS) ${ }^{40}$, we did not detect significant difference in neutral fat storage between seip-1 mutants and wild-type animals (Fig. 1b). The functionality of the SRS system was validated for its ability to detect elevated fat storage in daf-22 mutant animals, which are known to store more triglycerides ${ }^{41}$. We extended our analysis beyond neutral lipids by comparing wild-type and seip-1 mutant animals with a mass spectrometry-based lipidomic approach $^{42}$ (Supplementary Fig. 1b). The relative amount of two classes of lipids were significantly increased in seip-1(-) animals: phosphatidic acid (PA) and diacylglycerol (DAG) (Supplementary Fig. 1c). To investigate how such perturbation in lipid profile correlated with LD morphology, we used mRuby::DGAT-2 as a marker ${ }^{14}$. The median size of LDs in the intestinal cells was reduced from $0.81 \mu \mathrm{m}$ in diameter in wild-type animals to 0.42 $\mu \mathrm{m}$ in seip-1(-) animals (Fig. 1c, e). The alteration of LD size in seip-1(-) animals was reproducibly observed when we used DHS-3::GFP as an alternative LD marker (Supplementary Fig. $1 \mathrm{e}-\mathrm{g})^{43}$. Taken together, the abundance of small LDs correlated with the over-representation of membrane lipids with small head groups (PA and DAG), possibly because they are more compatible with high negative membrane curvature at junctions between the ER and nascent LDs that fail to expand in the absence of SEIP-1. Nevertheless, not all LDs were reduced in size in seip-1(-) animals (Fig. 1e). A similar phenotype of heterogeneous LDs was observed in seipin deficient budding yeast, Drosophila cells and mammalian fibroblasts ${ }^{30-32,34,44}$, suggesting that the dependence on seipin for LD homeostasis is evolutionarily conserved.

At the cellular level, energy balance is governed by fat storage in LDs and fat catabolism in peroxisomes and mitochondria. It has been reported that the yeast seipin ortholog Fld1 coordinates LD and peroxisome biogenesis at the ER, in conjunction with Pex3045. Interestingly, we observed an increase in the number of intestinal peroxisomes in seip-1(-) animals, when we used the peroxisomal thiolase DAF-22 as a marker (Fig. 1f, g). Therefore, SEIP-1 did not appear to be required for peroxisome biogenesis in C. elegans. Next, we visualized mitochondria using a red fluorescent protein that is targeted to the outer mitochondrial membrane (TOMM-20(1-54aa):mRuby). We did not detect gross morphological changes in mitochondria in seip-1(-) animals (Supplementary Fig. 1h-k). Our results suggest that loss of SEIP$1 /$ seipin function triggers secondary effects on fat catabolism in a species-specific manner.

SEIP-1 is enriched in peri-LD cages. To investigate how SEIP-1 affects LD size, we examined the sub-cellular localization of SEIP- 
a
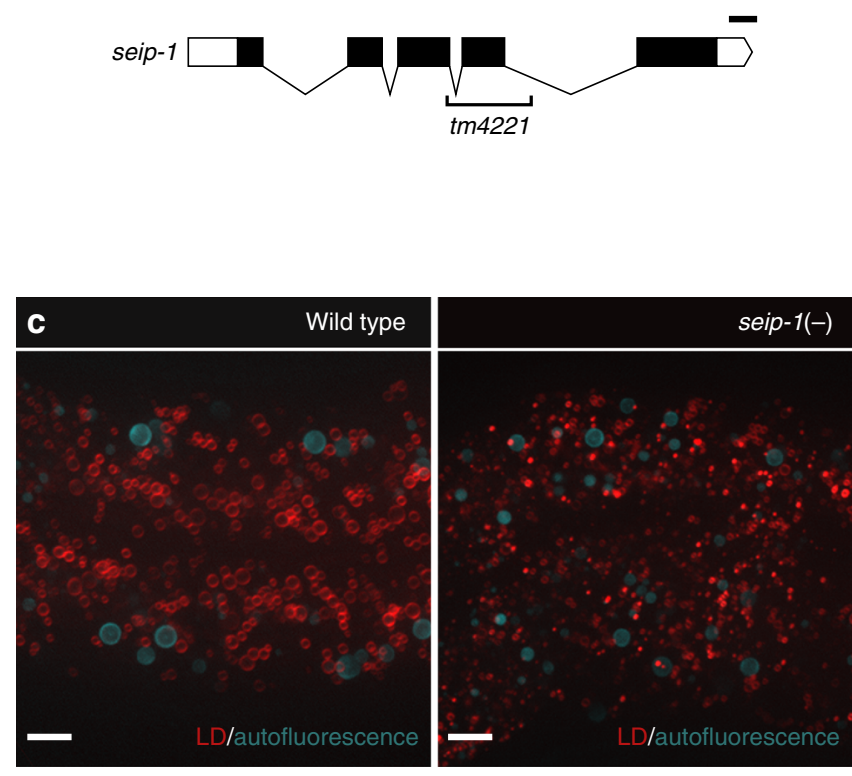

e

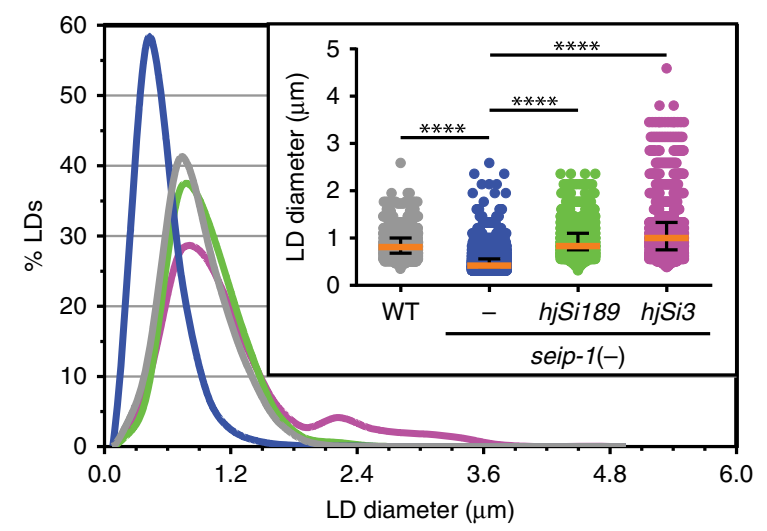

b

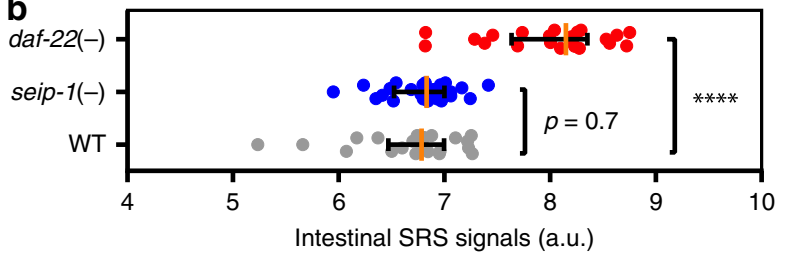

d

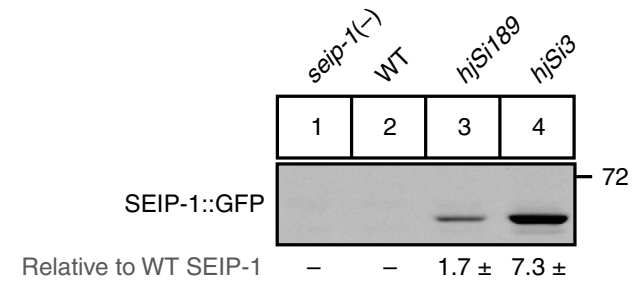

$0.9 \quad 2.8$

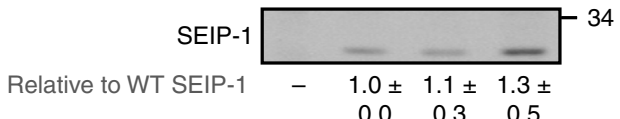

$\begin{array}{lll}0.0 & 0.3 & 0.5\end{array}$

Control

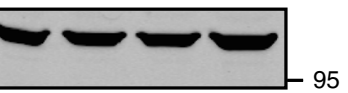

$\mathbf{f}$

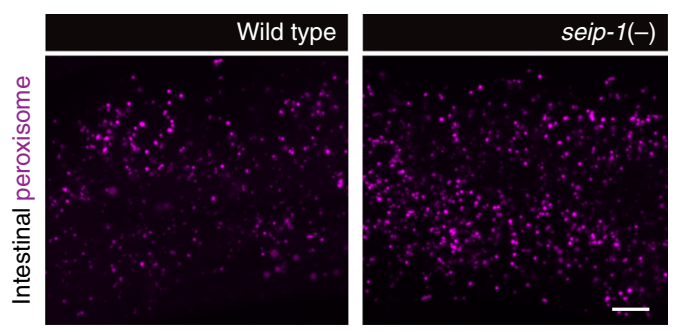

g

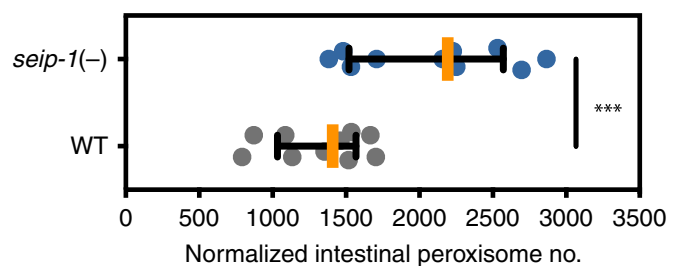

Fig. 1 SEIP-1 regulates LD size. a Schematic representation of seip-1. Exons and untranslated regions are indicated by black and white boxes, respectively. The region deleted in the $t m 4221$ allele is indicated. Scale bar $=100 \mathrm{bp}$. $\mathbf{b}$ Label-free quantification of neutral lipid content of individual animals by Stimulated Raman Scattering (SRS). Number of animals analyzed: wild type $(\mathrm{WT})=22$, daf-22(-) $=22$, seip- $1(-)=24$. c Visualization of LDs using mRuby::DGAT-2 (hjSi112) in a WT or seip-1 (tm4221) mutant larval L4 stage animal. mRuby is in red and autofluorescence from lysosome-related organelles (LROs) is pseudocolored cyan. A projection of $7.5 \mu \mathrm{m} z$ stack centering at the second intestinal segment is shown. $\mathbf{d}$ The expression levels of SEIP-1::GFP and endogenous SEIP-1 were determined by SDS-PAGE and immunoblotting with anti-SEIP-1 antibodies. A non-specific band at $\sim 95 \mathrm{kDa}$ served as a loading control. The expression level of endogenous SEIP-1 in WT animals served as a reference for normalization of signals in other samples. Mean \pm SD is presented from three independent experiments. e Frequency distribution of LD diameter. The curve was fitted twice using Fit Spline/LOWESS (20 points in smoothing window, 4000 segments) method based on a histogram with a bin size of $0.3 \mu \mathrm{m}$ (see Supplementary Fig. 1d, applicable to all subsequent frequency distribution curves). Inset: a scatter plot summarizing LD diameter. Total number of LDs measured: WT = 1680, seip-1 (tm4221) = 3934, hjSi189; seip-1 $(\mathrm{tm} 4221)=2224$, and hjSi3;seip-1 $(\mathrm{tm} 4221)=1209$. $\mathbf{f}$ Visualization of intestinal peroxisomes in a WT or seip-1 (tm4221) larval L4 stage animal. Signals from tagRFP::DAF-22 peroxisomal thiolase were pseudocolored magenta. A projection of $6.5 \mu \mathrm{m} z$ stack centering at the second intestinal segment is shown. $\mathbf{g}$ The total number of intestinal peroxisomes in a normalized volume of $80 \times 80 \times 6.5 \mu \mathrm{m}(x y z) . n=10$ animals. For all fluorescence images, scale bar $=5 \mu \mathrm{m}$. For all scatter plots, median with interquartile range is displayed. For all statistics, ${ }^{\star} p<0.05,{ }^{\star \star} p<0.01,{ }^{\star \star \star} p<0.001 ;{ }^{\star \star \star \star} p<0.0001$ (unpaired $t$-test), which applies to all subsequent statistical analysis

1 by expressing a SEIP-1::GFP fusion protein at the endogenous level from a single-copy transgene hjSi189, which is driven by the ubiquitous $d p y-30$ promoter (Fig. 1d). The hjSil89 transgene increased the median LD diameter of seip-1(-) animals to 0.83 $\mu \mathrm{m}$, which is similar to that in wild-type animals $(0.81 \mu \mathrm{m})$ (Fig. 1e). We concluded that the SEIP-1::GFP fusion protein was functional. In the C. elegans intestine, we observed SEIP-1::GFP exclusively in nanotubes that formed rings and cages around a subset of LDs (Fig. 2a). These nanotubes are most likely derived from ER tubules (Fig. 3e-g, Supplementary Fig. 3e-1). We named the SEIP-1::GFP positive structures peri-lipid droplet (peri-LD) cages. Similar observations were made when SEIP-1::tagRFP was expressed (Supplementary Fig. 2h). Furthermore, we used CRISPR to generate transgenic animals that express SEIP-1 with a $3 \times$ FLAG epitope tag at its C-terminus. Immunostaining revealed SEIP-1 positive signals around a subset of LDs (Supplementary 

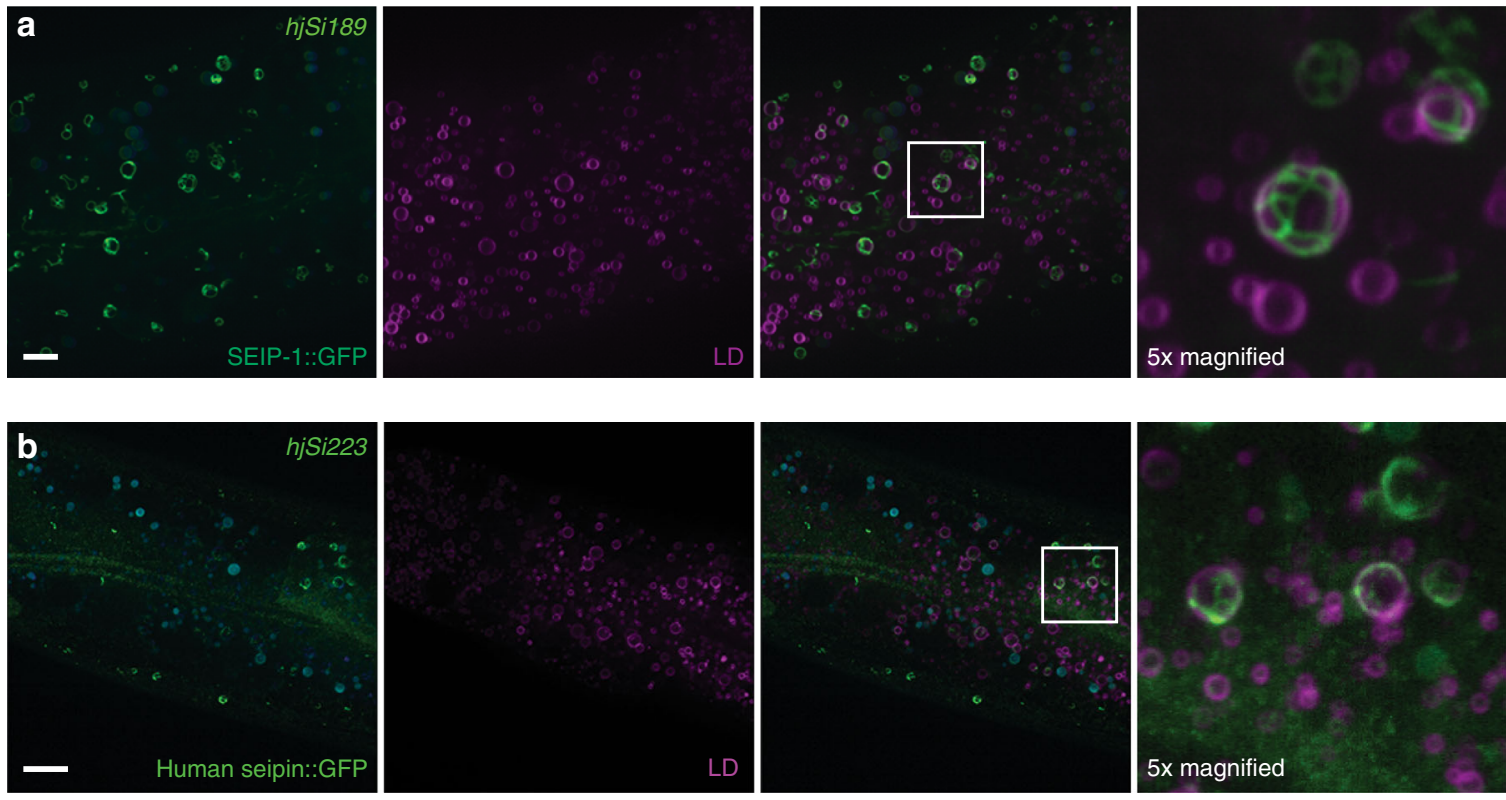

C
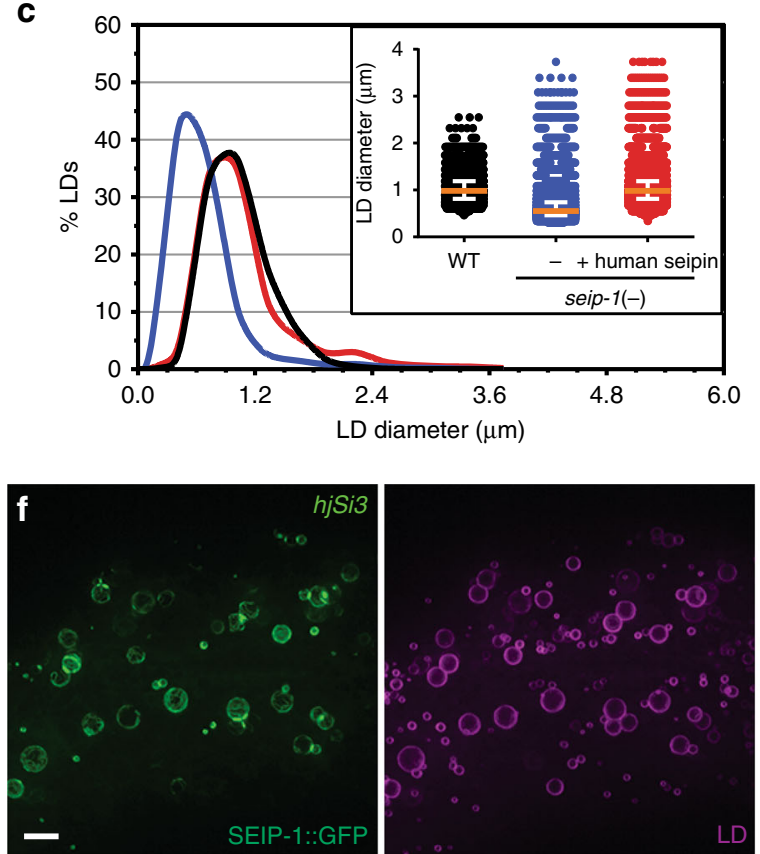

g
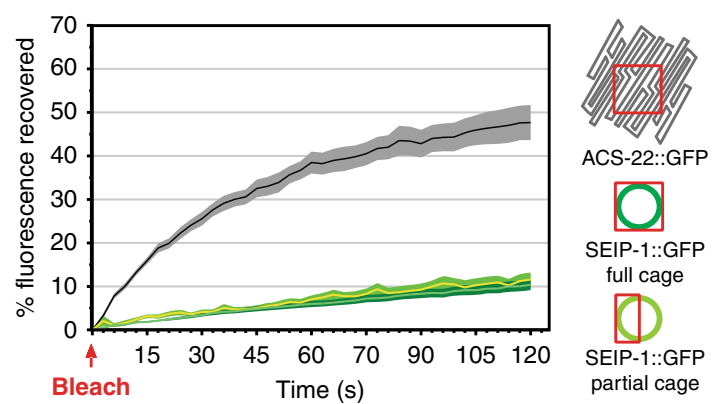

d
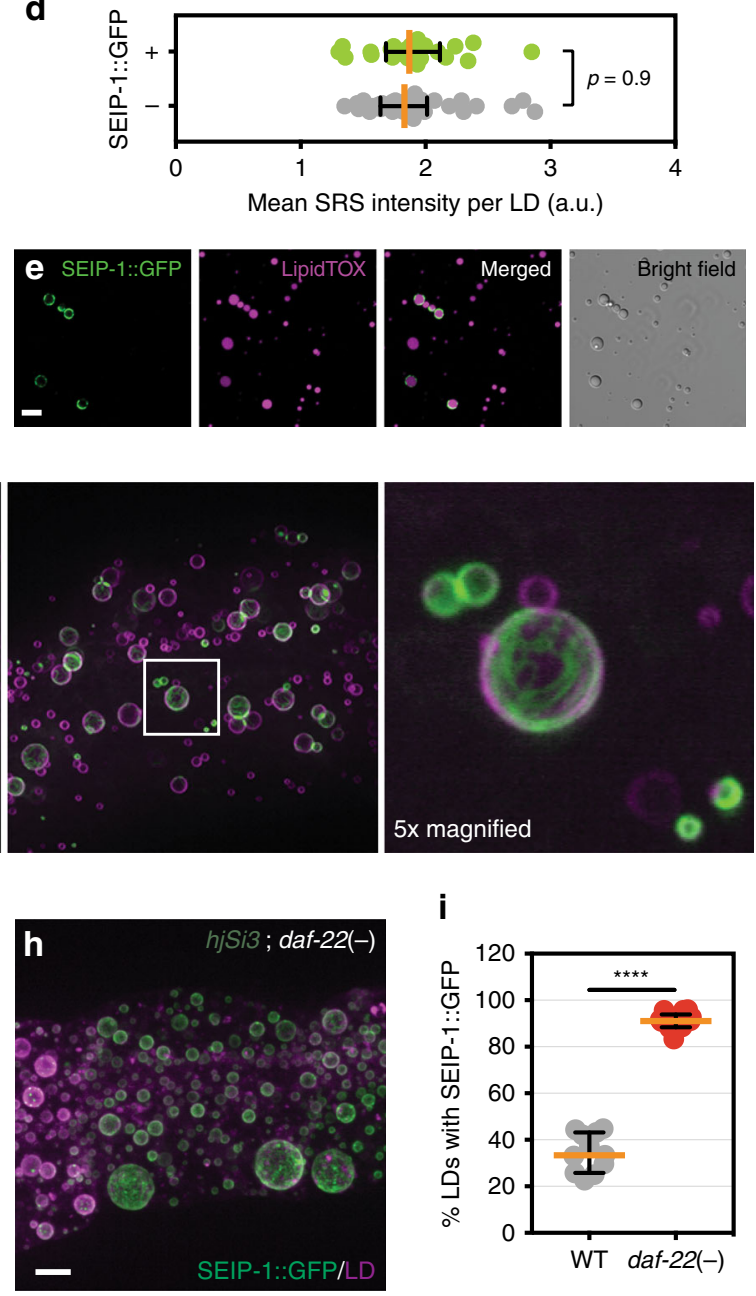

in tubular structures (Fig. 2b). The human seipin::GFP fusion protein was functional since it restored the median $\mathrm{LD}$ size of seip-1(-) animals to wild-type level (Fig. 2c). Taken together, the mechanism by which seipin family members are targeted to periLD cages is conserved.
Fig. 2a, b). Our results suggest that SEIP-1 exerts its effect at ER subdomains that are in close proximity to LDs.

Next, we expressed the human seipin protein fused to GFP from a single-copy transgene in C. elegans. Similar to C. elegans SEIP-1, human seipin was found to associate with a subset of LDs 
Fig. 2 SEIP-1 localizes to peri-LD cages. a Visualization of SEIP-1::GFP (hjSi189, lane 3 in Fig. 1d) in a larval L4 stage animal. Autofluorescence is pseudocolored blue. The LD marker mRuby::DGAT-2 (hjSi112) was used and the mRuby signal is pseudocolored magenta. A projection of $4.5 \mu \mathrm{m} z$ stack centering at the second intestinal segment is shown. The boxed area was magnified $5 \times$ and shown on the right. Scale bar $=5 \mu \mathrm{m}$. $\mathbf{b}$ Visualization of human seipin::GFP (hjSi223) in a seip-1 (tm4221) mutant larval L4 stage animal. The human seipin isoform 2 was expressed from a single-copy transgene. A projection of $3 \mu \mathrm{m} z$ stack centering at the second intestinal segment is shown. The LD marker and autofluorescence signals are displayed as in (a). The boxed area was magnified $5 \times$ and shown on the right. Scale bar $=10 \mu \mathrm{m}$. c Frequency distribution of LD diameter in larval L4 stage animals of indicated genotypes. The curve was fitted as in Fig. 1e. Inset: a scatter plot summarizing LD diameter. Total number of LDs measured: WT $=4600 ;$ seip-1(tm4221) $=$ 6069; seip-1 ( $t m 4221$ ); hjSi223 = 4258. $\mathbf{d}$ Label-free quantification of lipid concentration of individual LDs by SRS. LDs with a diameter of 0.8-1.7 $\mu$ m in the second intestinal segment were measured. Total number of LDs analyzed: SEIP-1::GFP $(-)=34$; SEIP-1::GFP $(+)=22$. e Visualization of SEIP-1::GFP in LDs purified from hjSi3 animals. LDs were stained with LipidTox Deep Red (pseudocolored magenta). $\mathbf{f}$ As in (a), but with an animal overexpressing SEIP-1::GFP (hjSi3, lane 4 in Fig. 1d). g Percentage of recovered fluorescence of ACS-22::GFP (resident ER protein, hjSi29) and SEIP-1::GFP (hjSi3) in wild-type animals. The connecting curves show the means and the filled areas show the SEM range. Number of photobleaching events: ACS-22::GFP =17; SEIP-1::GFP full cage $=15$; SEIP-1::GFP partial cage $=20$. $\mathbf{h}$ As in (f), but in daf-22(ok693) mutant background. $\mathbf{i}$ The percentage of LDs associated with SEIP-1::GFP in the second intestinal segment in wild-type and daf-22(ok693) mutant animals. Number of animals analyzed: wild type $($ WT) $=11$, daf-22(-) $=14$. For all scatter plots, median with interquartile range is displayed

Overexpression of SEIP-1 increased LD size. The seipin orthologs have been found at ER-LD junctions in mitotic cells $^{29,31,32}$. It has been proposed that the enrichment of seipin at these junctions promote the maturation of nascent $\mathrm{LDs}^{34}$. Our observations in $C$. elegans differentiated intestinal cells revealed sustained engagement of SEIP-1(+) structures with a subset of mature LDs. To probe the functional significance of such association, we generated a single-copy transgene ( $h j \mathrm{Si3})$ that overexpressed a SEIP-1::GFP fusion protein specifically in the $C$. elegans intestine (Fig. 1d). The localization of SEIP-1::GFP to peri-LD cages persisted in these animals. Using mRuby::DGAT-2 to mark all LDs, we determined that $\sim 40 \%$ of LDs were surrounded by peri-LD cages in each intestinal cell (Figs. $2 \mathrm{f}$ and $4 \mathrm{~g}$ ).

Opposite to the effect of loss of SEIP-1 activity on LD size, there was a significant increase in the median LD size in animals overexpressing SEIP-1::GFP (Fig. 1e). This was in part due to the appearance of LDs $>2 \mu \mathrm{m}$ in diameter, which were invariably associated with peri-LD cages. Nevertheless, SEIP-1 positive and negative LDs have similar concentration of neutral lipids, as determined by SRS (Fig. 2d). Taken together, we conclude that overexpression of SEIP-1::GFP protein conferred a gain-offunction phenotype: expansion of a subset of LDs.

Next, we purified LDs from hjSi3 transgenic animals by differential centrifugation and found that peri-LD cages labeled by SEIP-1::GFP remained stably associated with LDs (Fig. 2e). To further examine the spatial relationship between peri-LD cages with the ER tubular network, we generated animals that carry a second single-copy transgene that allowed the visualization of the ER lumen (signal sequence::mCherry::HDEL; hjSi158). Fluorescence signals from SEIP-1::GFP at the periphery of peri-LD cages juxtaposed with that from mCherry in the ER lumen (Supplementary Fig. 2i). However, the mobility of SEIP-1 was clearly different from a reference resident ER membrane protein, ACS22/acyl-coA synthetase ${ }^{14}$. Little recovery was observed when SEIP-1::GFP signals in whole or in part of cages were subjected to photobleaching (Fig. 2g). This was in contrast to the rapid recovery of ACS-22::GFP in the general ER network. Our biochemical and imaging results suggest that peri-LD cages are ER subdomains that preferentially associate with LDs. In addition, SEIP-1 is relatively immobile once incorporated into peri-LD cages, which may in part be due to regional differences of ER membrane constituents.

Peri-LD cages are ER subdomains. We sought to extend our observations by heterologous expression of SEIP-1 in mammalian cells. To this end, we generated COS7 cell lines that stably expressed a SEIP-1::Venus green fluorescent fusion protein at low levels, using the Sleeping Beauty transposon system ${ }^{46}$. Using endogenous protein disulfide isomerase (PDI) and TOM20 as ER and mitochondrial markers, respectively, we observed SEIP-1:: Venus in the ER and bright puncta that were diffraction-limited under basal conditions (Supplementary Fig. 3a). Addition of oleic acid, which promotes LD expansion, caused SEIP-1::Venus enrichment in structures that resembled peri-LD cages that were similar to those observed in C. elegans (Fig. 3a). To determine the temporal relationship of SEIP-1 enrichment and LD biogenesis, we generated additional COS7 cell lines that stably expressed low levels of SEIP-1::Venus and the LD biogenesis marker, LiveDrop $^{34}$. Time-lapse microscopy revealed the coincidence of SEIP$1::$ Venus and LiveDrop signals at puncta that progressively developed into LDs upon oleic acid loading (Fig. 3b, Supplementary Video 1). Finally, LDs marked by SEIP-1::Venus were significantly larger than those that were not marked in the same cells (Fig. 3c). Taken together, our results support a model that selective association of SEIP-1 with nascent LDs not only promotes their initial emergence from the ER but their subsequent expansion.

To study SEIP-1 localization by electron microscopy, we generated additional COS7 cell lines that stably expressed a SEIP1::APEX2 fusion protein at low levels ${ }^{47}$. The SEIP-1::APEX2 fusion protein could be detected around enlarged LDs, suggesting the function of SEIP-1 was preserved (Supplementary Fig. 3b-d). The APEX2 peroxidase reacts with diaminobenzidine (DAB) to generate local deposits that can be stained with uranyl acetate and osmium tetroxide. Accordingly, we found that SEIP-1::APEX2 was highly enriched on ER tubules in the proximity of LDs in oleic acid loaded cells (Fig. 3e-g, Supplementary Fig. 3e-l). The APEX2 mediated dark deposits were not detected in negative control cells that expressed SEIP-1::Venus (Fig. 3d). The SEIP-1:: APEX2 positive ER tubules showed similar morphology to those that are enriched with enzymes in the TAG biosynthetic pathways in Drosophila cells ${ }^{11}$. It has been proposed that such ER tubules constitute direct connections between ER and LDs. Our results suggest that peri-LD cages are conserved ER subdomains that support LD expansion.

Enrichment of SEIP-1 in peri-LD cages required polyunsaturated fatty acids. Is SEIP-1 enrichment in peri-LD cages sensitive to cellular metabolic activities? To address this question, we perturbed mitochondrial fission or fusion by RNAi against $d r p-1 /$ dynamin or $f z o-1 /$ mitofusin $^{48,49}$, respectively, and observed no significant effect on SEIP-1 targeting (Supplementary Fig. 2d-g). However, attenuation of peroxisomal fatty acid $\beta$-oxidation in daf-22 mutant animals caused promiscuous association of SEIP-1(+) peri-LD cages to almost all LDs (Fig. $2 \mathrm{~h}, \mathrm{i}$ ). Our results support a model that the accumulation of fatty acids 

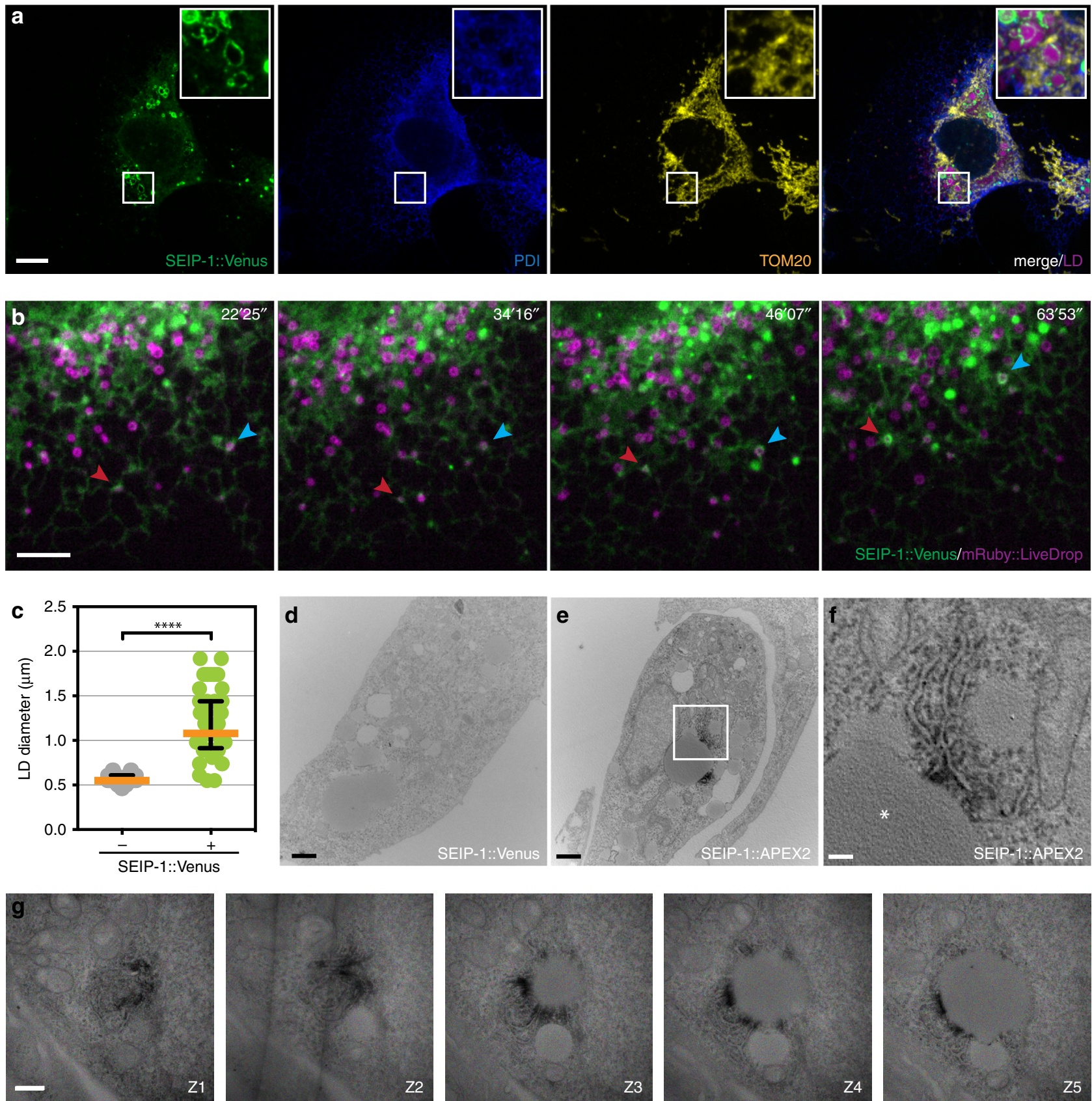

Fig. 3 Enrichment in peri-LD cages is an evolutionarily conserved property of seipin. a The SEIP-1::Venus protein was stably expressed in COS7 cells. The cells were incubated with oleic acid, fixed and immunostained for endogenous PDI (the ER) and TOM20 (mitochondria). Alexa Fluor (AF)-405 and 594 conjugated secondary antibodies were used for visualizing PDI and TOM20, respectively. AF594 signals were pseudocolored yellow. FAS was used to stain LDs and pseudocolored magenta. A projection of $4.5 \mu \mathrm{m} z$ stack is shown. The boxed area was magnified $3 \times$ and shown in the inset. Scale bar $=10 \mu \mathrm{m}$. b Time-lapse monitoring of peri-LD cages maturation. The SEIP-1::Venus protein and a nascent LD marker, mRuby::LiveDrop were stably co-expressed in COS7 cells. mRuby signals were pseudocolored magenta. The cells were incubated with oleic acid for $1.5 \mathrm{~h}$ before live imaging for another $1 \mathrm{~h}$ at $30-\mathrm{s}$ intervals. Arrows with the same color mark the same LiveDrop (+) LDs over time. Scale bar $=5 \mu \mathrm{m}$. c Quantification of LD size in COS7 cells stably expressing SEIP-1::Venus. A scatter plot of data from 48 LDs of each category, with median and interquartile range is shown. $\mathbf{d}$ Transmission electron micrograph of a COS7 cell stably expressing SEIP-1::Venus protein. The cell was incubated with oleic acid prior to fixation, and stained with diaminobenzidine (DAB), uranyl acetate, and osmium tetroxide prior to electron microscopy. No dark deposits were detected in this negative control. Scale bar $=1 \mu \mathrm{m}$. e As in (d), but with SEIP-1::V5-APEX2 fusion protein stably expressed. $\mathbf{f}$ The boxed area in (e) was magnified 5×. Dark deposits indicate the localization of SEIP-1::V5-APEX2 at ER tubules. The LD is indicated by an asterisk. Scale bar $=0.2 \mu \mathrm{m}$. $\mathbf{g}$ As in (e), but with five consecutive sections (150-nm thick) shown. Scale bar $=0.5 \mu \mathrm{m}$ 
a
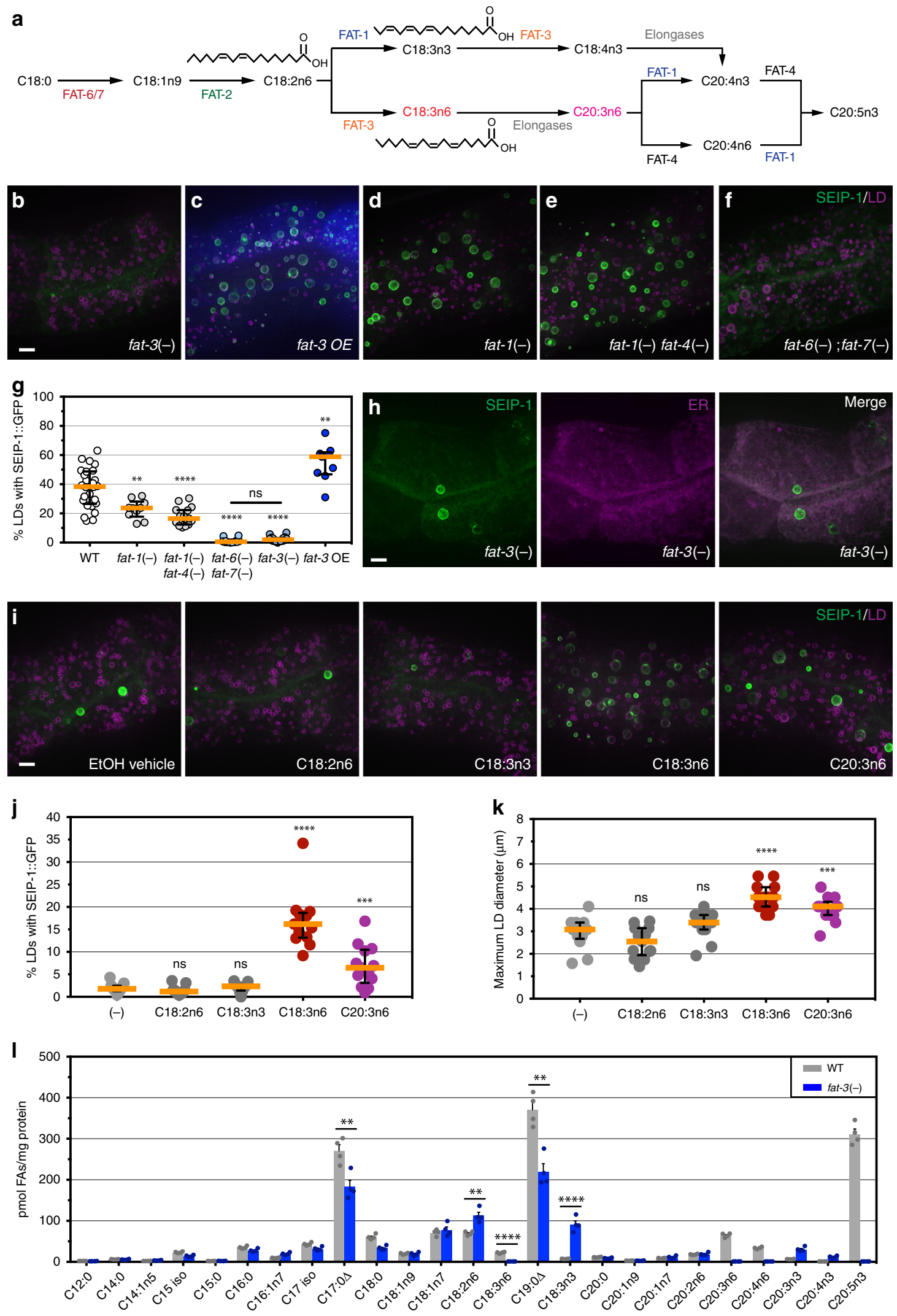

or their derivatives in daf-22 mutant animals promotes SEIP-1 enrichment in peri-LD cages, which in turn facilitates the continual expansion of LDs in these animals ${ }^{41}$.

To further elucidate the molecular mechanism for SEIP-1 enrichment in peri-LD cages, we mutagenized hjSi3 animals with the chemical mutagen ethyl methane sulfonate (EMS). From this forward genetic screen, we obtained two mutants (hj55 and hj56) in which SEIP-1::GFP was largely retained in the ER. Molecular cloning of these mutants identified mutations in the fat-3 gene, which encodes a fatty acid desaturase for the synthesis of 
Fig. 4 Mislocalization of SEIP-1::GFP in fat-3 mutant animals. a The biosynthetic pathway for polyunsaturated fatty acids in C. elegans. b Visualization of SEIP-1::GFP ( hjSi3) in a fat-3 (ok1126) mutant larval L4 stage animal. A projection of $4.5 \mu \mathrm{m} z$ stack centering at the second intestinal segment is shown. The LD marker mRuby::DGAT-2 (hjSi112) was used and the mRuby signal is pseudocolored magenta. c As in (b), but with a WT animal carrying a transgene that overexpressed FAT-3 (Ex[fat-3p::fat-3 genomic DNA::SL2::tagBFP]). The presence of blue fluorescence indicates the overexpression of FAT-3. d-f As in (b), but in fat-1(wa9), fat-1(wa9) fat-4(wa14), or fat-6(tm331);fat-7(wa36) mutant background. $\mathbf{g}$ A scatter plot summarizing the percentage of LDs associated with SEIP-1::GFP. Number of animals analyzed: WT $=26$, fat-3(-) $=18$, fat- $1(-)=10$, fat- $1(-)$ fat- $4(-)=15$, fat $-6(-) ; f a t-7(-)=12$, Ex $[$ fat $-3(+)]=9$. h As in (b), but with a luminal ER mCherry marker (hjSi158) in a fat-3 (ok1126) mutant animal. The mCherry signal is pseudocolored magenta. i Visualization of SEIP-1::GFP (hjSi3) and mRuby::DGAT-2 (hjSi112) in a fat-3(ok1126) mutant larval L4 stage animals grown in the presence of EtOH vehicle, linoleic acid (LA, C18:2n6), $\alpha$-linolenic acid (ALA, C18:3n3), $\gamma$-linolenic acid (GLA, C18:3n6) or dihomo- $\gamma$-linolenic acid (DGLA, C20:3n6) supplementation. A projection of $4.5 \mu \mathrm{m} z$ stack centering at the second intestinal segment is shown. $\mathbf{j}$ The effects of PUFAs supplementation on the percentage of LDs associated with SEIP1::GFP. Number of animals analyzed: $(-)=13, \mathrm{C} 18: 2 \mathrm{n} 6=13, \mathrm{C} 18: 3 \mathrm{n} 3=14, \mathrm{C} 18: 3 \mathrm{n} 6=13, \mathrm{C} 20: 3 \mathrm{n} 6=13$ (also applied to (k)). $\mathbf{k}$ As in (j), but the effects of PUFAs supplementation on the maximum LD diameter is shown. I The abundance of all fatty acids detected in WT or fat-3 (ok1126) larval L4 stage animals. Mean \pm SEM from four independent samples is shown. For all fluorescent images, scale bar $=5 \mu \mathrm{m}$. For all scatter plots, median with interquartile range is displayed

polyunsaturated fatty acids (PUFAs) in C. elegans (Fig. 4a) ${ }^{50}$. Specifically, FAT-3 introduces a double bond to C18:2n6 and $\mathrm{C} 18: 3 \mathrm{n} 3$ to yield $\mathrm{C} 18: 3 \mathrm{n} 6$ and $\mathrm{C} 18: 4 \mathrm{n} 3$, respectively. We noted that aberrant localization of SEIP-1::GFP was also observed in mutants that harbored a deletion in the fat-3 gene (ok1126) (Fig. 4b, h). This was not due to a change in the expression level of endogenous SEIP-1 and SEIP-1::GFP (Supplementary Fig. 4a), nor an overt change in LD size distribution of fat-3(-) animals (Supplementary Fig. 4f). We concluded that proper SEIP-1::GFP targeting is dependent on PUFAs that are direct or indirect products of the FAT-3 desaturase. Since FAT-3 does not colocalize with SEIP-1 and is distributed throughout the ER network (Supplementary Fig. 4i, j), it is plausible that additional mechanisms may be used to concentrate FAT-3 products in ER subdomains.

Based on previous reports, FAT-3 is required for the biosynthesis of multiple PUFAs ${ }^{50}$. This was confirmed using a quantitative gas chromatography-mass spectrometry (GC-MS) based assay (Fig. 41). Does SEIP-1::GFP enrichment in peri-LD cages require specific species of PUFAs? To address this question, we examined the localization of SEIP-1::GFP in animals that were deficient in other desaturases of the PUFAs biosynthetic pathway. FAT-1 and FAT-4 encode fatty acid desaturases that act upstream and downstream of FAT-3 (Fig. 4a). The percentage of LDs associated with SEIP-1::GFP was significantly reduced in fat-1 and fat-1 fat-4 mutant animals although the effect was not as pronounced as in fat-3 animals (Fig. $4 \mathrm{~d}, \mathrm{e}, \mathrm{g}$ ). Again, no change in the expression level of endogenous SEIP-1 and SEIP-1::GFP was observed (Supplementary Fig. 4a). We further considered three desaturases that produce FAT-3 substrates: FAT-2, FAT-6, and FAT-7. Intestinal specific RNAi against fat-2, fat-6, or fat7 significantly reduced the number of LDs that were associated with SEIP-1::GFP (Supplementary Fig. 4g, h). RNAi against fat-6 caused simultaneous reduction of fat -6 and fat -7 expression levels (Supplementary Fig. 6k). Accordingly, SEIP-1::GFP localization was disrupted partially in fat-6 or fat-7 single mutant animals (Supplementary Fig. 4c-e), but completely in fat-6; fat-7 double mutant animals (Fig. 4f, g). The latter animals also have a higher frequency of small $(0.6-0.9 \mu \mathrm{m})$ LDs than wild type (Supplementary Fig. 4f). We could not study SEIP-1::GFP localization of fat-2 mutant animals because of their larval arrest phenotype ${ }^{50}$. When FAT-3 was overexpressed, the percentage of LDs associated with SEIP-1::GFP was increased, suggesting that FAT-3 products play an instructive role in SEIP-1 targeting (Fig. 4c, g). Is a specific FAT-3 product required? To this end, we supplemented FAT-3 substrates (C18:2n6 and $\mathrm{C} 18: 3 \mathrm{n} 3)$ or products (C18:3n6 and C20:3n6) to the E. coli diet of fat-3(-) animals. The enrichment of SEIP-1::GFP in peri-LD cages was significantly increased by $\mathrm{C} 18: 3 \mathrm{n} 6$ and C20:3n6 supplementation, while C18:2n6 or
C18:3n3 had no effect (Fig. 4i, j). This was accompanied by an increase in the maximal LD diameter in C18:3n6 and C20:3n6 treated animals (Fig. 4k), consistent with the role of SEIP-1 in promoting LD expansion. Taken together, our genetic and dietary supplementation experiments indicate that proper targeting of SEIP-1::GFP is at least partially dependent on specific PUFAs.

Enrichment of SEIP-1 in peri-LD cages required microbial fatty acids. Our quantitative GC-MS analysis revealed that in addition to a reduction of PUFAs, the abundance of two cyclopropane fatty acids (CFAs), C17:0 $\Delta$ and C19:0 $\Delta$, was also significantly reduced in fat-3(-) animals (Fig. 4l). Since CFAs are strictly derived from E. coli and are not synthesized by C. elegans, our results implied that fat-3(-) animals are partially defective in absorbing dietary microbial fatty acids. CFAs are synthesized by the CFA synthase from monounsaturated fatty acids (Supplementary Fig. 5a $)^{51}$. They are major constituents of bacterial phospholipids that are important for membrane integrity under stress $^{52}$. We next asked if the mislocalization of SEIP-1::GFP in fat-3(-) animals was in part due to a reduction of diet-derived CFAs. To this end, we obtained the E. coli CFA synthase deletion mutant and its parental strain BW25113 from the Keio collection $^{53}$. Using GC-MS, we confirmed that the $\Delta c f a$ strain indeed was unable to synthesize C17:0 $\Delta$ and C19:0 $\Delta$ (Supplementary Fig. 5b). Next, we fed hjSi3; hjSi112 transgenic animals that expressed SEIP-1::GFP and mRuby::DGAT-2 with BW25113 and $\Delta c f a$ E. coli. Using mRuby::DGAT-2 as a pan-LD marker in the intestine, we noted a reduction of median $\mathrm{LD}$ diameter and total LD volume in animals fed $\Delta c f a E$. coli (Fig. 5c, d). Furthermore, the percentage of LDs that are associated with SEIP-1::GFP was also significantly reduced (Fig. 5e). Quantitative GC-MS analysis indicated an almost complete loss of C17:0 $\Delta$ and $\mathrm{C} 19: 0 \Delta$, and a concomitant increase of $\mathrm{C} 16: \ln 7$ and $\mathrm{C} 18: \ln 7$ in the fatty acid profile of worms fed $\Delta c f a E$. coli (Fig. $5 f$ ). This was consistent with C16:1 and C18:1n7 being the precursors of C17:0 $\Delta$ and C19:0 $\Delta$ in E. coli, respectively (Supplementary Fig. 4a, b). Based on our model, residual targeting of SEIP-1::GFP to peri-LD cages in fat-3 $(-)$ animals could be attributed to reduced, but measurable levels of CFAs. Indeed, complete disruption of SEIP-1::GFP targeting ensued when fat-3(-) animals were fed $\Delta c f a$ E. coli (Supplementary Fig. $5 \mathrm{c}$ ). Our results demonstrate that $C$. elegans obtain CFAs from their microbial diet and incorporate them efficiently into their cellular lipids. Such dietary CFAs have a significant impact on fat content, LD morphology and SEIP-1 targeting.

PUFAs and CFAs regulate SEIP-1 targeting in mammalian cells. To test the effect of PUFAs and CFAs on SEIP-1 targeting in a simplified, heterologous system, we used the previously 

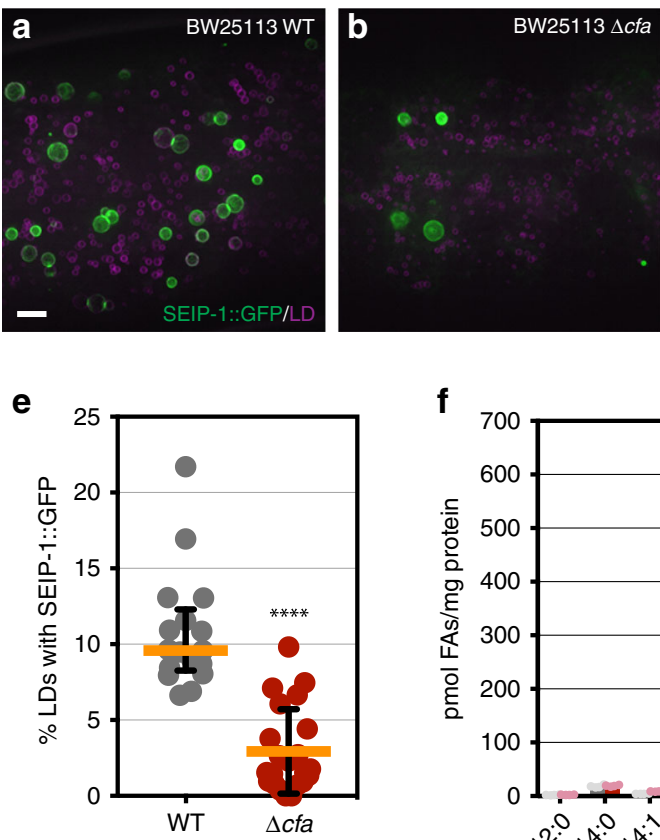

f
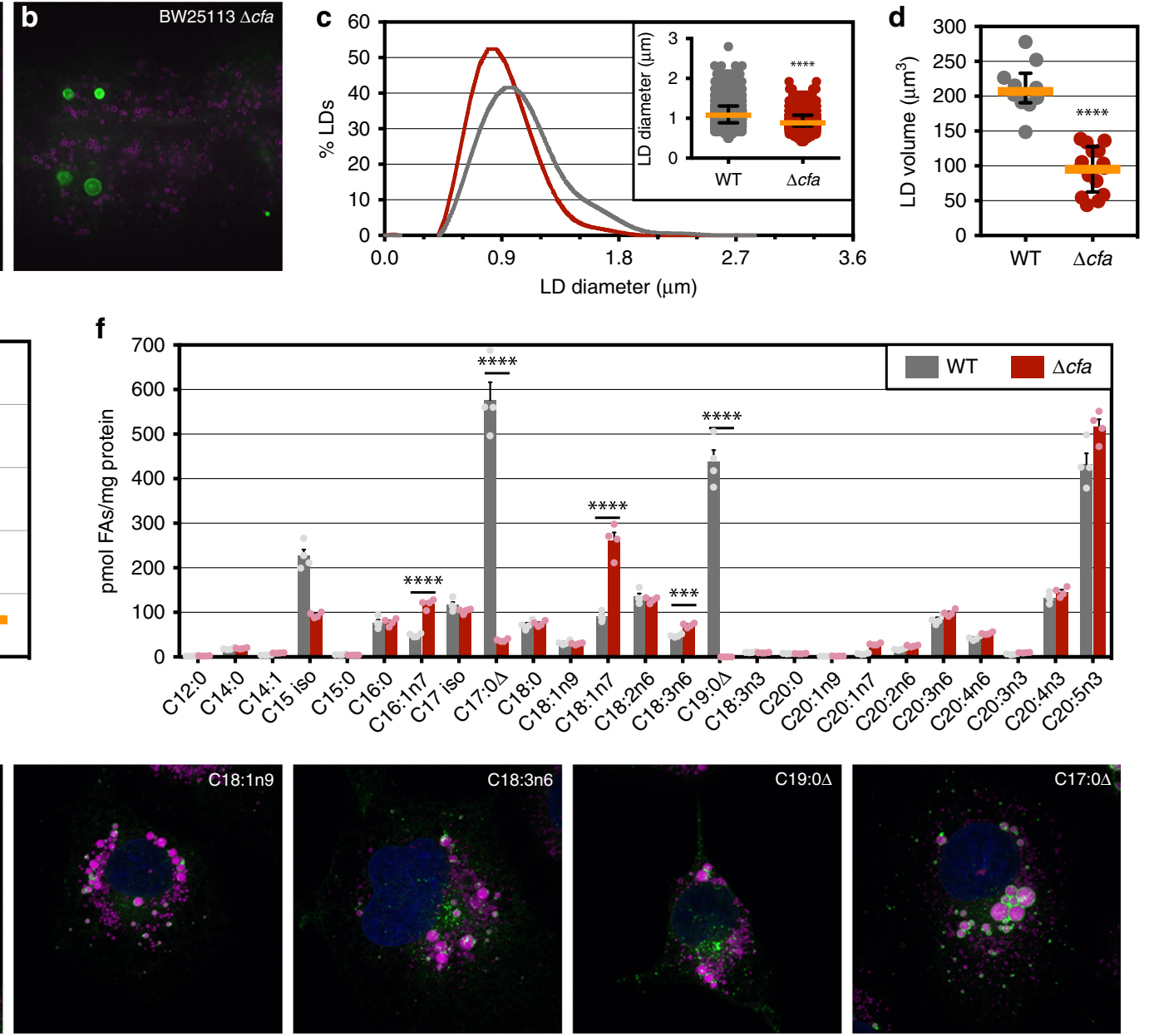

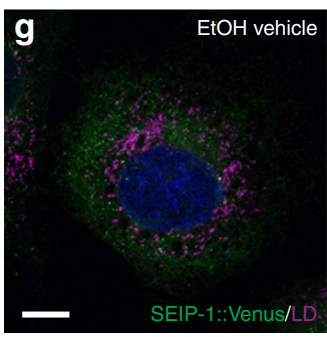

h

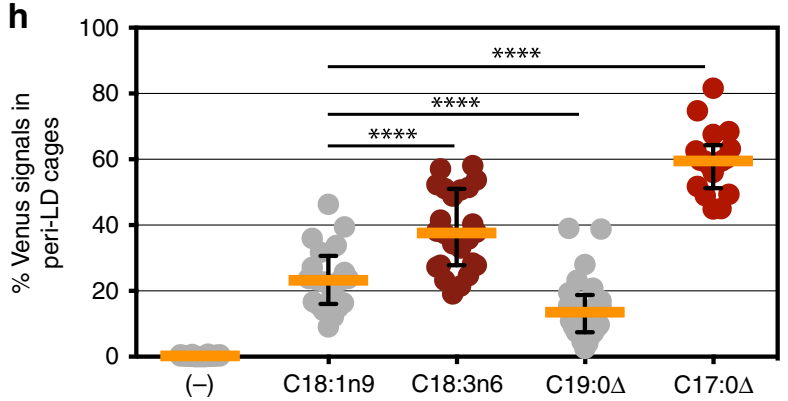

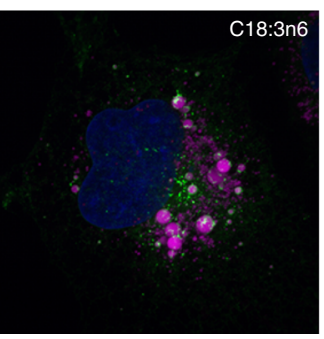
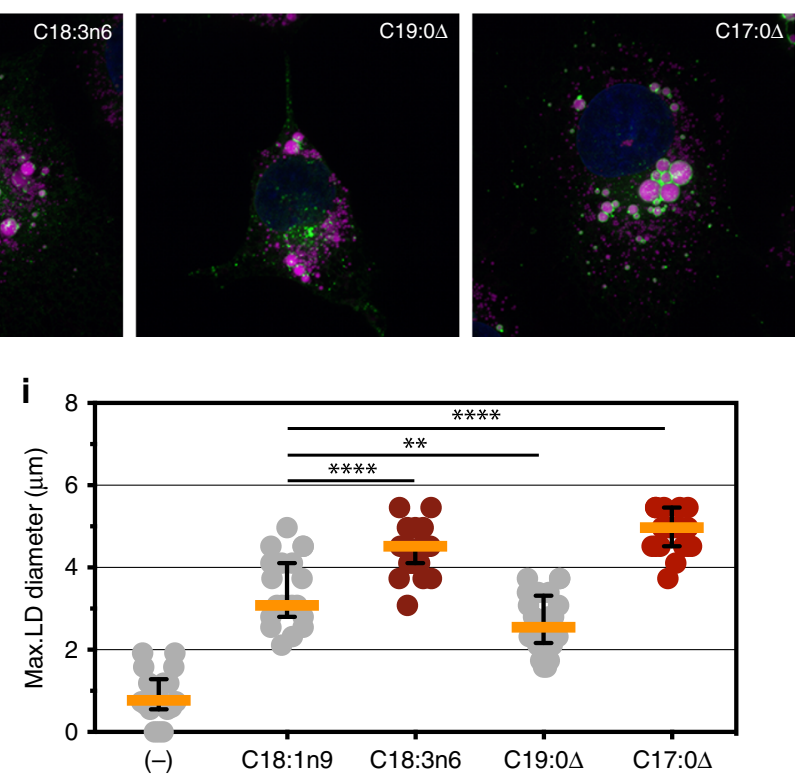

Fig. 5 Bacterial cyclopropane-FAs regulate SEIP-1::GFP targeting. a Visualization of SEIP-1::GFP (hjSi3) in a larval L4 stage animal fed WT E. coli BW25113. A projection of $4.5 \mu \mathrm{m} z$ stack centering at the second intestinal segment is shown. The LD marker mRuby::DGAT-2 (hjSi112) was used and the mRuby signal is pseudocolored magenta. Scale bar $=5 \mu \mathrm{m}$. b As in (a), but with an animal fed $\Delta$ cfa mutant E. coli BW25113. c Frequency distribution of LD diameter in larval L4 stage animals that were fed wild-type (gray) or $\Delta c f a$ (red) E. coli. The curve was fitted as in Fig. 1e. Inset: a scatter plot summarizing LD diameter. Total number of LDs measured: BW25113 WT fed $=2414 ; B W 25113 \Delta$ cfa fed $=2704$. d A scatter plot summarizing the total LD volume in the second intestinal segment of each animal. Number of animals analyzed: WT $=10, \Delta c f a=15$. e The lack of dietary CFA reduced the percentage of LDs associated with SEIP-1::GFP. Number of animals analyzed: WT $=17, \Delta c f a=22$. $\mathbf{f}$ The abundance of all fatty acids detected in worms fed wild-type (gray) or $\Delta c f a$ (red) E. coli. Mean \pm SEM from four independent samples is shown. $\mathbf{g}$ COS7 cells stably expressing SEIP-1::Venus were supplemented with either EtOH vehicle, oleic acid (OA, C18:1n9), $\gamma$-linolenic acid (GLA, C18:3n6), 11,12-methylene octadecanoic acid (C19:0 $\Delta$ ), or 9,10-methylene hexadecanoic acid (C17:0 $\Delta$ ). Cells were incubated with $100 \mu \mathrm{M}$ FAs for $24 \mathrm{~h}$. LDs were stained with LipidTox Red (pseudocolored magenta) and nuclei were stained with Hoechst 33342 (blue). A projection of $4.5 \mu \mathrm{m}$ z stack is shown. Scale bar $=10 \mu \mathrm{m}$. $\mathbf{h}$ The effect of FA supplementation on the percentage of SEIP-1::Venus signals at peri-LD cages. Number of cells analyzed: $(-)=18, C 18: 1 n 9=20, C 18: 3 n 6=24, C 19: 0 \Delta=24, C 17: 0 \Delta=18$ (also applied to (i)). $\mathbf{i}$ As in (h), but the effect of FA supplementation on the maximum LD diameter is shown. For all scatter plots, median with interquartile range is displayed

generated COS7 cell lines that stably expressed a SEIP-1::Venus fusion protein at low levels. In comparison with oleic acid (C18:1n9), supplementation of $\mathrm{C} 18: 3 \mathrm{n} 6$ and $\mathrm{C} 17: 0 \Delta$, but not C19:0 $\Delta$ had significantly stronger effects on promoting LD expansion, which correlated with SEIP-1::Venus enrichment in
peri-LD cages (Fig. 5g-i, Supplementary Fig. 5g, h). Furthermore, the effect of C17:0 $\Delta$ was dose-dependent (Supplementary Fig. $5 \mathrm{~d}-\mathrm{f}$ ). Our results suggest that polyunsaturated C18:3n6 and microbial C17:0 play conserved roles in regulating SEIP-1 targeting in mammalian cells. 

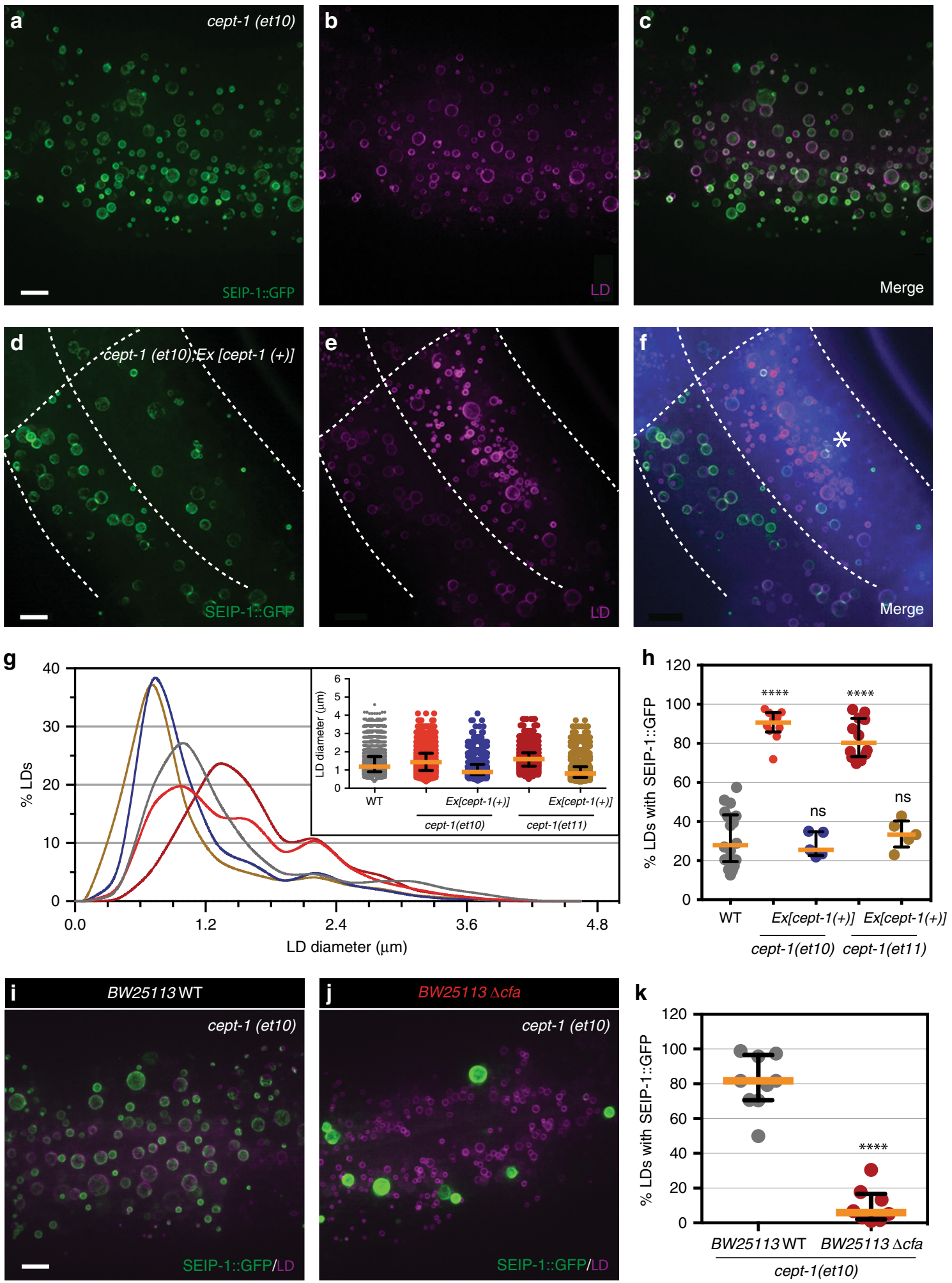

Attenuation of phosphatidylcholine synthesis enhances SEIP-1 targeting to peri-LD cages. Phospholipids with PUFAs and CFAs as acyl chains may shape the membrane microenvironment of the ER. Therefore, we used an intestine-specific RNAi strain to knockdown a number of genes that encode phospholipid biosynthetic enzymes in C. elegans, in order to identify cell-autonomous, membrane-structural regulators of SEIP-1 targeting. We found that intestine-specific depletion of pcyt-1 and cept-1 but not cept-2 caused promiscuous association of SEIP-1(+) peri-LD cages with almost all LDs (Supplementary Fig. 6a). PCYT-1 and CEPT-1 act sequentially in the Kennedy pathway for phosphatidylcholine (PC) synthesis in C. elegans ${ }^{54}$, suggesting that SEIP-1 targeting is sensitive to cellular PC content. Our RNAi results could be recapitulated in mutant animals that carried two different cept-1 loss-of-function alleles ${ }^{55}$ (Fig. 6a-c, h). These animals also showed an increase in the median LD size (Fig. 6g). Overexpression of CEPT-1 restored SEIP-1 targeting to peri-LD cages around a subset of LDs and 
Fig. 6 Attenuation of phosphatidylcholine synthesis promotes SEIP-1::GFP targeting to peri-LD cages. a Visualization of SEIP-1::GFP (hjSi3) in a cept-1(et10) mutant larval L4 stage animal. A projection of $4.5 \mu \mathrm{m} z$ stack centering at the second intestinal segment is shown. b As in (a), but with a mRuby::DGAT-2 LD marker (hjSi112). The mRuby signal is pseudocolored magenta. c As in (a), but with GFP and mRuby signals merged. d As in (a), but with a cept-1(et10) mutant animal carrying a rescuing transgene (Ex[vha-6p::cept-1a cDNA::SL2::tagBFP]). The polycistronic transgene enabled the co-expression of CEPT-1 and tagBFP. The white dotted lines approximate the cell boundaries. e As in (d), but with a mRuby::DGAT-2 LD marker (hjSi112). The mRuby signal is pseudocolored magenta. f As in (d), but with GFP, mRuby and tagBFP signals merged. Expression of CEPT-1, as indicated by blue fluorescence in the cell marked with an asterisk, restored differential targeting of SEIP-1::GFP to peri-LD cages surrounding a subset of LDs. $\mathbf{g}$ Frequency distribution of LD diameter in larval L4 stage animals with indicated genetic backgrounds. The curve was fitted as in Fig. 1e. Inset: a scatter plot summarizing LD diameter. Total number of LDs measured: WT $=2347$; cept-1(et10) =1306; cept-1(et10);Ex[cept-1(+)] =854; cept-1(et11) =1319; cept-1(et11);Ex[cept-1(+)] = 936. h The percentage of LDs associated with SEIP-1::GFP. Number of animals analyzed: WT $=18$, cept-1(et10) $=10$, cept-1(et10); Ex [cept-1(+)] =5, cept-1(et11) $=12$, cept-1(et11);Ex[cept-1(+)] =5. i As in (a), but fed with WT E. coli BW25113. j As in (a), but fed with $\Delta$ cfa mutant E. coli BW25113. k The percentage of LDs associated with SEIP-1::GFP in cept-1(et10) animals fed different $E$. coli diets. Number of animals analyzed: WT $=9, \Delta c f a=8$. For all fluorescent images, scale bar $=5 \mu \mathrm{m}$. For all scatter plots, median with interquartile range is displayed

reduced the median LD size of these mutant animals (Fig. $6 \mathrm{~d}-\mathrm{h}$ ). Interestingly, the cept-1 loss-of-function phenotypes could be suppressed by withdrawing dietary CFAs (Fig. 6i-k) or by blocking FAT-3 or FAT-6 mediated PUFAs synthesis (Supplementary Fig. 6e-j). We conclude that the precise control of CFAs, PUFAs, and PC levels is necessary for maintaining SEIP-1 dependent LDs diversity.

SEIP-1 enrichment in peri-LD cages is partially dependent on COPII. SEIP-1 is an integral membrane protein. We therefore asked if the canonical secretory pathway is involved in the enrichment of newly synthesized SEIP-1 in peri-LD cages. To this end, we used the Sleeping Beauty transposon system to generate COS7 cell lines that constitutively expressed low levels of SEIP-1:: Venus. In addition, these cells could be induced to express a GTPlocked, dominant negative (DN) form of SAR1a(H79G)::mRuby by doxycycline (DOX). The expression of SAR1a(DN)::mRuby effectively retarded COPII-dependent membrane trafficking, without altering the SEIP-1::Venus expression level (Supplementary Fig. 7a). However, SEIP-1::Venus was not found in periLD cages upon SAR1a(DN) induction (Fig. 7a and Supplementary Fig. 7b, c). Accordingly, we observed significantly enhanced colocalization of SEIP-1::Venus and SAR1a(DN)::mRuby signals, indicating the retention of SEIP-1 to the ER (Fig. 7b). This was accompanied by a decrease in the colocalization of SEIP-1::Venus with signals from the fluorescent LD dye, $\mathrm{FAS}^{56}$ (Fig. 7c). Timelapse microscopy indicated that SEIP-1::Venus puncta failed to elaborate into peri-LD cages upon oleic acid loading, when SAR1a (DN) was induced (Supplementary Fig. 7d, Supplementary Video 2). In C. elegans, the targeting of SEIP-1 to peri-LD cages was partially impaired when $s e c-23$, which encodes an ER exit site component, was knocked down by RNAi in the intestine (Fig. 7d-f). It has been shown that COPII-decorated ER exit sites (ERES) are in close proximity of LDs, for the delivery of specific LD proteins ${ }^{57}$. It is plausible that COPII plays a related role in SEIP-1 targeting.

\section{Discussion}

In this paper, we combined genetic, biochemical, light and electron microscopy approaches to demonstrate the preferential enrichment of SEIP-1/seipin to an ER subdomain, termed the peri-LD cage. Peri-LD cages selectively engage a subset of nascent LDs and promote their subsequent expansion. Recruitment of SEIP-1 to peri-LD cages demands PUFAs and microbial CFAs. More specifically, $\gamma$-linolenic acid (C18:3n6) and cyclopropane fatty acid $(\mathrm{C} 17: 0 \Delta)$ have the strongest effect on SEIP-1 targeting in $C$. elegans and mammalian cells. Our results suggest that the heterogeneity of LDs can be enforced not only by LD proteins, but through inter-organelle contacts with the ER.
How does the enrichment of SEIP-1 in peri-LD cages increase LD size? In the energy-replete state, a balance of lipolysis and TAG synthesis determines LD size ${ }^{11,58}$. Attenuation of lipolysis, i.e., blocking the release of fatty acids from triglycerides in LDs, will therefore increase LD size. Lipolysis is rate-limited by the recruitment of adipose triglyceride lipase (ATGL) to the LD surface $^{59}$. We have previously shown that the C. elegans ATGL ortholog (ATGL-1) is found at $\mathrm{LDs}^{41}$. Since the ATGL-1::GFP fusion protein was observed at the surface of LDs with or without peri-LD cages (Supplementary Fig. 2j), we concluded that peri-LD cages did not impede lipolysis by blocking ATGL-1 recruitment. Development of assays for measuring the lipolytic rate at the single LD level will be necessary to probe additional effects of peri-LD cages on lipolysis.

The de novo synthesis of triglycerides (TAG) is dependent on LD associated DGAT2. In mammalian cells, DGAT2 transits from the ER to the LD surface upon lipid loading ${ }^{11,19}$. In $C$. elegans, the DGAT2 ortholog (DGAT-2) associates stably with LDs $^{14}$. Throughout this study, mRuby::DGAT-2 was used to mark all LDs and we did not observe enhanced DGAT-2 residency at LDs with peri-LD cages. Nevertheless, it is plausible that peri-LD cages can increase the local concentration of additional proteins that are rate-limiting for TAG synthesis. These proteins should be well conserved since peri-LD cage association increased LD size in both C. elegans and mammalian cells. For example, the glycerol-3-phosphate acyltransferase 4 (GPAT4), which acts upstream of DGAT2 in the TAG synthesis pathway, is also known to migrate from the ER to LDs and functionally couple with seipin ${ }^{11,60}$. Although the mammalian GPAT4 shares sequence homology with a number of $C$. elegans acyltransferases, its functional ortholog awaits to be identified definitively.

In budding yeast, several proteins, including Ldb16 and Ldo45, act with the seipin ortholog Fld1 at ER-LD junctions to regulate LD size ${ }^{61-66}$. In addition, human Promethin/TMEM159, a remote ortholog of Ldo45, has been shown to associate with $\operatorname{LDs}^{67,68}$. However, BLAST homology searches for their orthologs in C. elegans did not return significant hits. Since the conserved central loop region of seipin orthologs is flanked by divergent $\mathrm{N}$ and C-termini, it is tempting to speculate that seipin orthologs may have conserved and species-specific partners that modulate LD morphology in a convergent manner.

Cellular membranes are in large part composed of phospholipids that consist of a head group and two fatty acyl chains. The combination of saturated, monounsaturated, and polyunsaturated acyl chains in phospholipids modulates the packing and curvature of membranes that contribute to organelle identity and function ${ }^{36,69}$. Although humans obtain PUFAs from their diet, PUFAs are synthesized endogenously by a set of desaturases in C. elegans ${ }^{50}$. Based on biochemical and molecular dynamics simulations, PUFAs have the unique ability to support the formation of highly curved and 
a

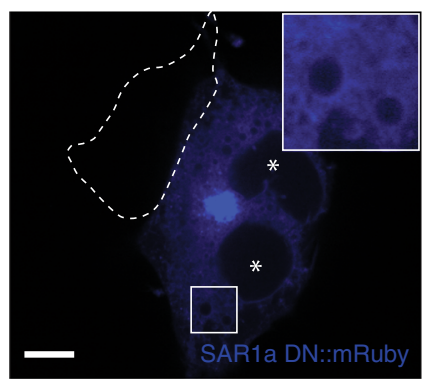

$\mathrm{OA} 6 \mathrm{~h} \longrightarrow \mathrm{SAR} 1 \mathrm{a}$ DN induction $+\mathrm{OA} 12 \mathrm{~h}$
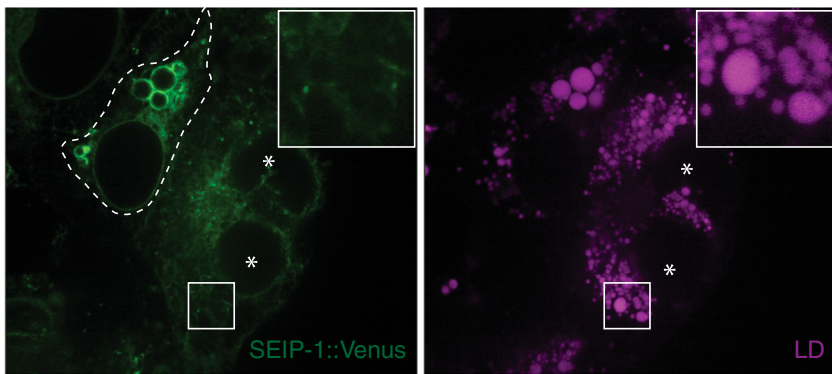

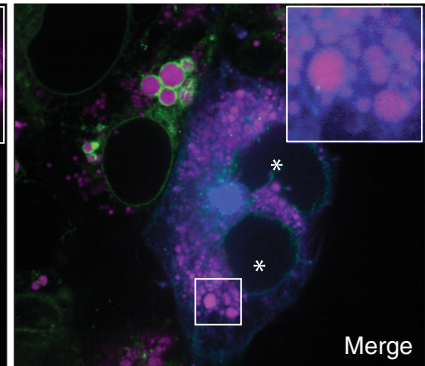

b

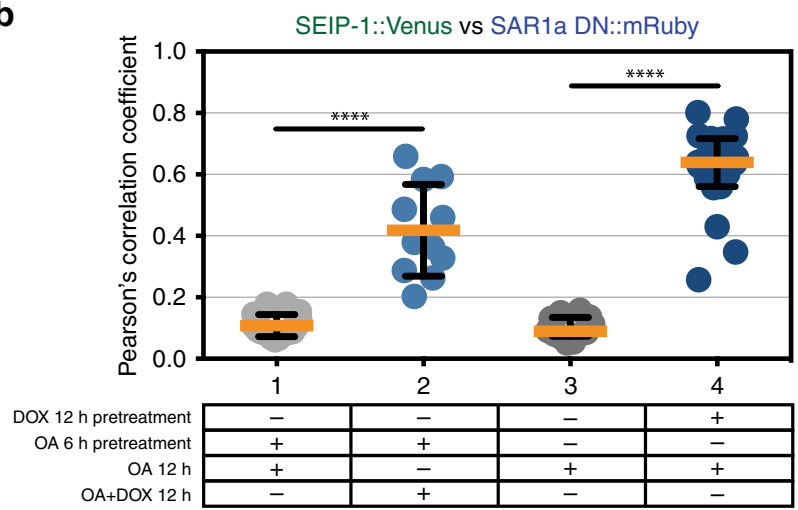

C

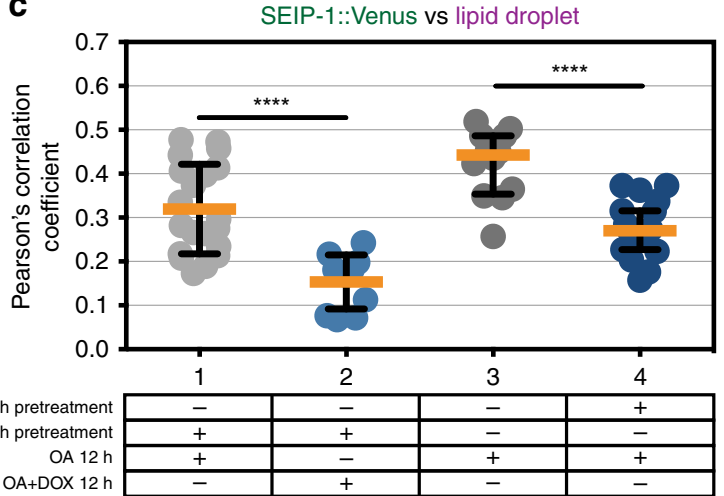

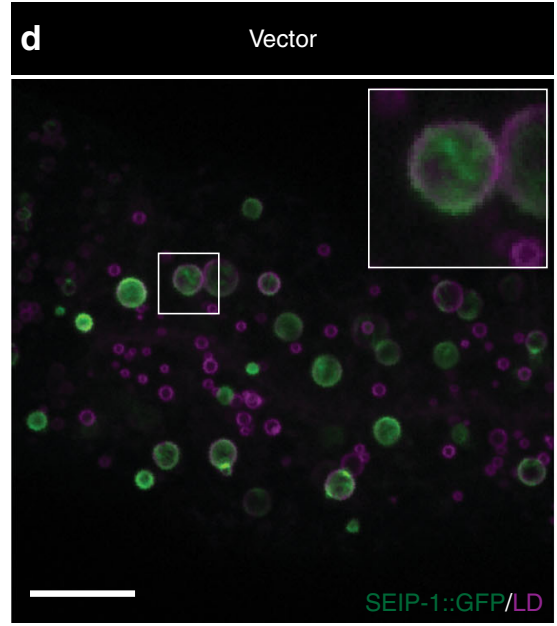

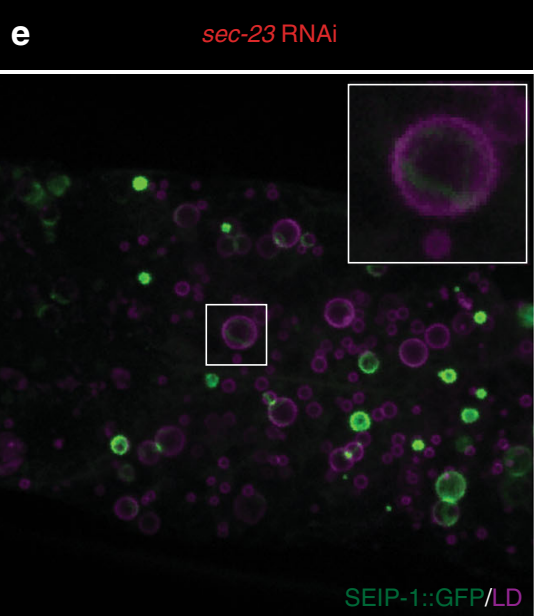

f SEIP-1::GFP vs mRuby::DGAT-2

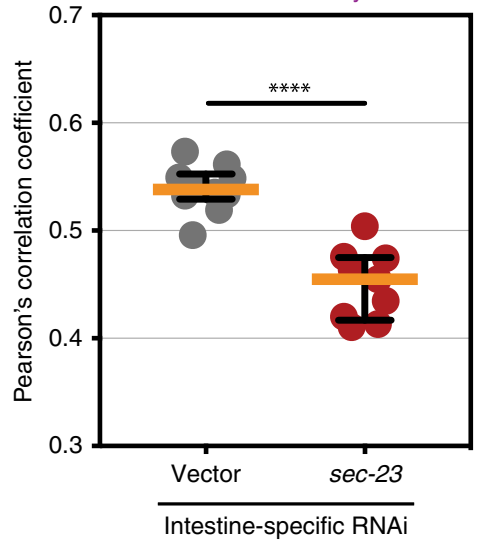

Fig. 7 Inhibition of COPII perturbs the proper targeting of SEIP-1. a Doxycycline (DOX)-inducible overexpression of SAR1a(H79G)::mRuby (dominantnegative (DN) mutant) in COS7 cells that stably expressed SEIP-1::Venus. Cells were pre-loaded with $400 \mu \mathrm{M}$ oleic acid (OA) for $6 \mathrm{~h}$ and induced by $1 \mu \mathrm{g} / \mathrm{mL}$ DOX for $12 \mathrm{~h}$ in the presence of OA. Lipid droplets were stained by FAS and pseudocolored magenta, mRuby signal is pseudocolored blue. Boxed regions were magnified $3 \times$ and shown in the inset. Single focal planes are shown. Asterisks mark the cells that overexpressed SAR1a DN mutant, while the adjacent cells with relatively low level of induction are outlined by dashed lines. Scale bar $=10 \mu \mathrm{m}$. $\mathbf{b}$ Colocalization coefficient of SAR1a DN::mRuby and SEIP-1::Venus signals, calculated from $4.5 \mu \mathrm{m}$ z-stack volumes. Number of cells analyzed: column $1=20$, column $2=11$, column $3=12$, column $4=19$ (also applied to (c)). Columns 1 and 3 serve as controls to columns 2 and 4, respectively. Images in (a) were quantified and summarized in column 2; images in Supplementary Fig. 7b were quantified and summarized in column 4. c As in (b), but with the colocalization coefficient of SEIP-1::Venus and FAS (LD dye) signals calculated. d Visualization of SEIP-1::GFP (hjSi3) with control RNAi knockdown in a larval L4 stage animal. A LD marker mRuby::DGAT-2 (hjSi112) was used and the mRuby signal is pseudocolored magenta. Projections of $4.5 \mu \mathrm{m} z$ stack centering at the second intestinal segment are shown. The boxed region is magnified $3 \times$ and shown in the inset. Scale bar $=10 \mu \mathrm{m}$. e As in (d), but with intestine-specific RNAi knockdown of sec-23. f Colocalization coefficient of SEIP-1::GFP and mRuby::DGAT-2 signals calculated from $4.5 \mu \mathrm{m}$ z-stacked volumes in the second intestinal segment. Number of animals analyzed: vector $=10, \sec -23=9$. For all scatter plots, median with interquartile range is displayed

densely packed membranes ${ }^{70}$. It is plausible that ER tubules that constitute peri-LD cages are composed of such membranes since SEIP-1 was immobile once incorporated into peri-LD cages (Fig. 2g). This could be due to the tight fitting of fatty acyl chains around SEIP-1. PUFAs in peri-LD cages may also act as diffusion barriers to additional proteins, such as those that support LD expansion. Our work supports the idea that PUFAs have fundamental structural roles in defining membrane territories.

It has been reported that Fld1/seipin and Pex30 define an ER subdomain for $\mathrm{LD}$ and peroxisome biogenesis in yeast ${ }^{45,71}$. Therefore, we were intrigued by the possibility that Pex30 could be used to label SEIP-1(+) peri-LD cages in heterologous systems. 
To this end, we generated COS7 cell lines that stably co-expressed SEIP-1::Venus and Pex30::mRuby. Under basal conditions, no significant colocalization of the two proteins was observed (Supplementary Fig. 8a). However, induction of LD expansion by oleic acid loading prompted the co-enrichment of SEIP-1 and Pex30 in peri-LD cages (Supplementary Fig. 8b). Interestingly, time-lapse microscopy revealed that the formation of SEIP-1:: Venus puncta preceded Pex30::mRuby puncta at the initial phase of oleic acid loading (Supplementary Fig. 8c, Supplementary Video 3). It is tempting to speculate that the sequential enrichment of SEIP- 1 and Pex30 reflects stepwise assembly of additional lipid or protein components of peri-LD cages.

There is an increasing appreciation on how the host metabolism can be modulated by the microbiome, in part through microbial metabolites ${ }^{72,73}$. Our work reveals cyclopropane fatty acids (CFAs, C17:0 $\Delta$, and C19:0 $\Delta$ ) as new players in hostmicrobe interaction. Cyclopropane fatty acyl chains are generated through the action of CFA synthase on monounsaturated precursors in membrane phospholipids of E. coli and other bacteria ${ }^{51}$. Since there is no eukaryotic CFA synthase, the detection of CFAs in C. elegans indicates their effective absorption and incorporation into host cellular lipids. CFAs have also been detected in human adipose tissue where seipin is strongly expressed 22,37 . Nevertheless, it was hitherto unclear how CFAs play a role in host metabolism. Our work hinted at one possible mechanism: CFA-dependent protein targeting.

In summary, our results reveal unexpected and conserved roles of microbial and polyunsaturated fatty acids on cellular fat storage. They modulate SEIP-1/seipin targeting to peri-LD cages and in turn promote LD expansion in C. elegans and mammalian cells. The selective association of peri-LD cages to a subset of LDs also strengthen the notion of LD heterogeneity ${ }^{9}$. Distinct populations of LDs may carry specific cargos beyond neutral lipids. They may be subject to differential turnover during fed or fasted states. Our results implicate the selective deployment of dietary and endogenous fatty acids in defining organelle identity through specialized membrane environments.

\begin{abstract}
Methods
Strains and transgenes. The wild-type C. elegans strain was Bristol N2. All experimental animals were maintained at $20^{\circ} \mathrm{C}$. The following alleles were obtained from the Caenorhabditis Genetics Center (CGC), which is funded by NIH Office of Research Infrastructure Programs (P40 OD010440): LG III, unc-119(ed3); LG X, cept-1(et10), cept-1(et11); LG IV, fat-1 fat-4(wa9 wa14), fat-3(ok1126); LG V, sid-1(qt78). The seip-1(tm4221) allele was obtained from Dr. Shohei Mitani (National Bioresource Project for the nematode). The following transgenes or CRISPR-generated alleles were used: hjSi3[vha-6p::seip-1 cDNA::GFP_TEV_3xFLAG::let-858 $\left.3^{\prime} U T R\right]$ II, hjSi56[vha-6p::3xFLAG_TEV_GFP::F59A1.10 coding::let$8583^{\prime} U T R$ ] IV, hiSil12[vha-6p::3xFLAG_TEV_mRuby2::dgat-2 cDNA::let-858 3' UTR] IV, hjSi127[vha-6p::mRuby2::CB5::let-858 $\left.3^{\prime} U T R\right]$ IV, hjSi158[vha-6p::SEL-1 (1-79aa)::mCherry::HDEL::let-858 3'UTR] I, hiSi189[dpy-30p::seip-1 cDNA:: GFP_TEV_3xFLAG::tbb-2 $\left.3{ }^{\prime} U T R\right]$ II, hjSi222[fat-3p::fat-3 genomic DNA::GFPTEV-3xFLAG::fat-3 $\left.3^{\prime} U T R\right]$ I, hjSi223[dpy-30p::human seipin isoform 2 cDNA (codon-optimized)::GFP_TEV_3xFLAG::tbb-2 $\left.3^{\prime} U T R\right]$ II, hiSi434[dpy-30p::seip-1 cDNA::tagRFP::tbb-2 $3^{\prime}$ UTR] II, hjSi489[dpy-30p::tomm-20(1-54aa)::mRuby] II, hjSi494[vha-6p::sid-1 cDNA::dhs-28 3'UTR] I, hjEx21, hjEx22[vha-6p::cept-1a cDNA_SL2_tagBFP2::let-858 3'UTR], hjEx23[fat-3p::fat-3 genomic DNA_SL2_tagBFP2::fat-3 3'UTR, dhs-3(hj120) [dhs-3::GFP_3xFLAG], seip-1(hj196) [seip-1:: TEV_3xFLAG], daf-22(hj234) [tag-RFP_3xFLAG::daf-22].
\end{abstract}

C. elegans genetic screen. To isolate mutant animals with altered localization of SEIP-1::GFP, we mutagenized $h j S i 3$ animals with ethyl methane sulfonate (EMS) using standard procedures. We screened $\sim 38,000$ haploid genomes and selected mutant F2 animals on a UV fluorescence dissecting microscope (Leica). Mutant animals were back-crossed with $h j S i 3$ animals at least twice and their phenotype was subsequently verified by confocal microscopy. For molecular cloning, we focused on one complementation group comprised of $h j 55$ and $h j 56$ alleles. Genetic mapping with the Hawaiian isolate CB4856 placed both alleles on LGIV. Molecular lesions in the fat-3 gene were identified by whole-genome sequencing (Illumina) and verified by targeted Sanger sequencing. The $h j 55$ and $h j 56$ alleles encode the mutations Gln325Stop and Gly329Asp, respectively. The fat-3(ok1126) deletion allele, outcrossed four times with $\mathrm{N} 2$, was subsequently used as the reference strain The mislocalization of SEIP-1::GFP in the hjSi3; fat-3(ok1126) strain could be rescued by a fat-3p::fat-3 genomic DNA::SL2::tagRFP::fat-3 3'UTR transgene.

RNA interference-based knockdown in C. elegans. RNA interference (RNAi) was performed based on published methods ${ }^{73}$. The RNAi vectors for knocking down fat-2, fat-6 or fat-7 were taken from the Ahringer library and transformed into OP50 [rnc14::DTn10 laczgA::T7pol camFRT]. The RNAi vectors for knocking down $d r p-1$, fzo-1, pcyt-1, cept-1, and cept-2 were constructed for this study and transformed into the above OP50 strain. Primers used for molecular cloning: $d r p-1$, 5'-TCAACAGAATATTCTCGACAACAAAC- ${ }^{\prime}$ and $5^{\prime}$-TTCAAATGAGACTTC TGGTACG-3'; fzo-1, 5' -CTTTCTCCAGAATTCGATAGC-3' and 5' -ATACAATG CTCAAAATGTCG-3'; pcyt-1, 5'-CTGTAACTTACAGCTGGCCG-3' and 5'-CGT TCAAACCTCCGTCCTTATG-3'; cept-1, 5'-CCAGTCTTCGTCACATTGAAC-3' and $5^{\prime}$-CGTCGCAATCCATAACAAGAC-3'; cept-2, 5'-GCTACGCTTTGCAAT TAGG-3' and 5'-CAATAAATGCCCATATGGTCG-3'. Fresh overnight cultures of OP50 RNAi clones were seeded on NGM plates with $0.4 \mathrm{mM} \mathrm{IPTG}$ and $100 \mu \mathrm{g} / \mathrm{mL}$ Ampicillin. The seeded plates were incubated at room temperature for 1 day. Six to eight L4 larval P0 animals of each genotype were picked onto the plates and removed after 2 days. F1s were imaged at the L4 larval stage. To achieve intestinespecific knockdown, we generated a single-copy transgene that expressed wild-type SID-1 protein under the control of intestine-specific vha-6 promoter (hjSi494). The transgene was then crossed into sid-1(qt78) mutant animals ${ }^{74}$. hjSi3 [SEIP-1::GFP] and hjSil12 [mRuby::DGAT-2] were subsequently introduced into hjSi494;sid-1 ( $q 778)$ and used for RNAi-based knockdown following the above procedures.

Fluorescence imaging of $\boldsymbol{C}$. elegans and mammalian cells. Fluorescence images of L4 larval animals or mammalian cells were acquired on a spinning disk confocal microscope (AxioObeserver Z1, Carl Zeiss) equipped with a piezo Z stage using a $\times 100$, numerical aperture (NA) 1.46 oil Alpha-Plan-Apochromat objective, on an Neo sCMOS camera (Andor) controlled by the QQ3 software (Andor). For GFP, a $488 \mathrm{~nm}$ laser was used for excitation and signals were collected with a 500-550-nm emission filter. For mCherry and tagRFP, a $561 \mathrm{~nm}$ laser was used for excitation and signals were collected with a 580.5-653.5-nm emission filter. For autofluorescence from lysosome-related organelles, a $488 \mathrm{~nm}$ laser was used for excitation and signals were collected with a 580.5-653.5-nm emission filter. For tagBFP, a $405 \mathrm{~nm}$ laser was used for excitation and signals were collected with a 417-477-nm emission filter.

Optical sections were taken at $0.5 \mu \mathrm{m}$ intervals and z stacks of images were exported from iQ3 to Imaris 8 (Bitplane) for processing and 3D reconstruction. Ten focal planes in total ( $4.5 \mu \mathrm{m}$ in $z$ axis) were used for all projected fluorescence images unless otherwise indicated. For worm intestine, the diameter of each LD present in the second intestinal segment was all manually fitted using the spot function in Imaris. Pearson correlation coefficients were calculated in Imaris without thresholding.

To quantify enrichment of SEIP-1::Venus signals at peri-LD cages upon FAs loading, FA supplementation was performed blindly (i.e., the identity of FAs loaded to each plate was not known to the experimenter who took the images and analyzed the data). Ten optical sections at $0.5 \mu \mathrm{m}$ intervals of each cell were exported as raw greyscale images from Imaris and subsequently reconstituted in ImageJ as maximum signal projections. For each projection, a mean background value was initially calculated and used as the threshold. The intensity of Venus signals at all peri-LD cages to that in the overall cell area was calculated using the ROI function in ImageJ and used to assess the enrichment of SEIP-1::Venus at periLD cages.

Maintenance of mammalian cells. COS7 cells (ATCC, CRL-1651) were cultured in DMEM (Gibco) with 10\% FBS (Gibco) and 1\% antibiotic-antimycotic (Gibco) in humidified incubators flowed with $5 \% \mathrm{CO}_{2}$.

FAs supplementation in mammalian cells. Supplementation was performed based on a published method ${ }^{75}$. In short, the $100 \mathrm{mM}$ stock solution of each FAs was diluted immediately into the complete medium pre-heated to $60^{\circ} \mathrm{C}$. Medium was then equilibrated to $37^{\circ} \mathrm{C}$ before loading. To induce $\mathrm{LD}$ expansion, cells were incubated with $400 \mu \mathrm{M}$ FAs for $12 \mathrm{~h}$ unless otherwise indicated.

Time-lapse live cell imaging. Cells were initially seeded in 35-mm MatTek glassbottom dishes coated with poly-D-lysine. Upon OA loading, cells were imaged in a stage-top incubator that was flowed with humidified air with $5 \% \mathrm{CO}_{2}$, and held at $37^{\circ} \mathrm{C}$ by a CU-501 temperature controller (Chamlide $\mathrm{TC}^{\mathrm{TM}}$ ). Time-lapse images were acquired using the above confocal system in 30-s intervals controlled by a definitive focus ( $\mathrm{fc12}$, Zeiss) unless otherwise indicated.

Fluorescence recovery after photobleaching (FRAP) assay. Photobleaching was performed on larval L4 stage animals using the spinning disk confocal microscope described above. The region of interest (ROI) was chosen to cover the entire or half peri-LD cage in hjSi3 animals. For each FRAP event, the size of the ROI was kept constant for both background and reference regions. One or more ROIs of at least 
eight animals were examined for each strain. Normalized fluorescence intensity (NI) was calculated using the following formula, taking background fluorescence (Back) and fluorescence in an unbleached reference ROI (Ref) into consideration.

$$
N I_{\text {ROI } t 1}=\left(\frac{I_{\text {ROI } t 1}-I_{\text {Backt } 1}}{I_{\text {ROI } t 0}-I_{\text {Backto }}}\right) *\left(\frac{I_{\text {Ref } t 0}-I_{\text {Back } t 0}}{I_{\text {Ref } t 1}-I_{\text {Backt1 }}}\right)
$$

Label-free lipid imaging in C. elegans. For analyzing single lipid droplets, an integrated femtosecond (fs) stimulated Raman scattering (SRS) and two-photon excited fluorescence (TPEF) microscope system was used for label-free imaging of LDs in C. elegans ${ }^{40}$. L4 larval animals ( $h j S i 3$ ) were dissected in the physiological buffer $\left(150 \mathrm{mM} \mathrm{NaCl}, 3 \mathrm{mM} \mathrm{KCl}, 2 \mathrm{mM} \mathrm{MgCl} 2,3 \mathrm{mM} \mathrm{CaCl}_{2}, 10 \mathrm{mM}\right.$ glucose and $15 \mathrm{mM}$ HEPES; $340-345 \mathrm{mmol} / \mathrm{kg}$ ) containing $0.2 \mathrm{mM}$ levamisole and mounted on a $2 \%$ agarose pad. Raw signals from GFP, SRS and tryptophan were acquired sequentially from a field of view (FOV) of $30 \times 30 \mu \mathrm{m}$ in z stacks of $7 \mu \mathrm{m}$ at $0.5 \mu \mathrm{m}$ intervals. For quantification, an averaged background noise $\left(\overline{I_{\text {noise }}}\right)$ from photodiode and lock-in amplifier (LIA) was measured and SRS signals of glycerol $\left(I_{\text {glycerol }}\right)$ in the same FOV were acquired for normalization prior to acquisition of the GFP, SRS, and tryptophan signals. LD in dissected hjSi3 animals that was $0.8-1.7 \mu \mathrm{m}$ in diameter and present in focal plane $1-8$ ( $3.5 \mu \mathrm{m}$ in $z$ axis $)$ was used for quantification. For each LD in each focal plane, total intensity of normalized SRS signals $\left(I_{\text {plane }}\right)$ was calculated by:

$$
I_{\text {plane }}=\sum_{i=1}^{n} \frac{I_{\text {raw } i}-\overline{I_{\text {noise }}}}{I_{\text {glycerol } i}-\overline{I_{\text {noise }}}}
$$

where $I_{\text {raw } i}$ is the intensity of $i$ th pixel present in the LD and $n$ is the number of all pixels enclosed in the LD. $I_{\text {plane }}$ from all focal planes that each LD spanned were summed up as the total intensity of each $\mathrm{LD}\left(T I_{\mathrm{LDs}}\right)$. Volume of each $\mathrm{LD}$ was calculated using Image in raw SRS images.

For whole-worm lipid measurement, live L4 larval animals were directly immersed in the physiological buffer containing $0.2 \mathrm{mM}$ levamisole and mounted on a $8 \%$ agarose pad. For each animal, a projection image with maximal SRS intensity was acquired using a picosecond SRS microscope system as previously developed equipped with a $\times 20$ air objective (Plan-Apochromat, $0.8 \mathrm{NA}$, Zeiss) ${ }^{76}$ Quantification was performed according to published protocols ${ }^{77}$. Briefly, raw total intestinal SRS intensity of each animal was adjusted by $\overline{I_{\text {noise }}}$ and $I_{\text {glycerol }}$ as described above, and subsequently averaged by the total intestinal pixel number. At least 20 animals were imaged and quantified for each genotype.

Antibodies and western blotting for $\boldsymbol{C}$. elegans. Antibodies against SEIP-1 were raised by injection of the peptide ${ }^{261}$ KKEEPGLLDLRKRK, corresponding to the Cterminus of SEIP-1, into rabbits (YenZym). Antibodies were purified against the antigen immobilized on the AminoLink Plus Coupling resin (Pierce, \#44894) and used in western blotting at a dilution of 1:100. IRDye 800CW donkey anti-rabbit secondary antibodies (LI-COR) were used at 1:5000. Fluorescence signals were visualized using an Odyssey Infrared Imaging system (LI-COR) and analyzed using Odyssey software according to manufacturer's instructions. Background signals were measured from multiple areas of the blot without proteins, averaged, and subtracted from integrated intensity of each protein band. The non-specific signal from a $\sim 95 \mathrm{kDa}$ protein was used as the loading control unless otherwise indicated. An uncropped scan of the western blot in Fig. 1d is shown in Supplementary Fig. 9.

LDs isolation from C. elegans. LDs isolation from C. elegans was performed using a published $\operatorname{method}^{78}$. In brief, around 100,000 synchronized L4 larval animals were washed and resuspended in buffer A (20 mM Tricine, $250 \mathrm{mM}$ sucrose, $\mathrm{pH}$ 7.8, $0.5 \mathrm{mM}$ PMSF). The worm resuspension was dounced on ice in a $15 \mathrm{~mL}$ Tenbroeck tissue grinders (Pyrex) for 60 initial times and subsequently transferred to a $15 \mathrm{~mL}$ Potter-Elvehjem tissue grinder (Wheaton) for douncing 80 more times. Worms debris and post-nuclear supernatant (PNS) was removed by centrifuge at $1000 \times g$ for $15 \mathrm{~min}$. The resulted supernatant was overlaid with buffer B $(20 \mathrm{mM}$ HEPES, $100 \mathrm{mM} \mathrm{KCl}, 2 \mathrm{mM} \mathrm{MgCl}_{2}, \mathrm{pH}$ 7.4) at equal ratio and used for gradientultracentrifugation in SW41Ti swinging bucket rotor (Beckman-Coulter). LDs were collected at buoyant fractions after a 2-h centrifugation at 10,000 $\times g$.

For fluorescence imaging, $10 \mu \mathrm{LD}$ sample was diluted with $90 \mu \mathrm{l}$ buffer B and incubated with 1:1000 diluted LipidTox Deep Red (Thermo Fisher, \#H34477) for $30 \mathrm{~min}$ on ice. Fluorescence images were acquired using a confocal microscope (Olympus, FV1000).

Dietary supplementation of polyunsaturated fatty acids (PUFAs). Pure PUFAs: linoleic acid (LA), $\alpha$-linolenic acid (ALA), $\gamma$-linolenic acid (GLA), and dihomo- $\gamma$-linolenic acid (DGLA) (Nu-Chek Prep) were dissolved in absolute ethanol at a final concentration of $50 \mathrm{mM}$ as stock solutions. Each stock solution was freshly diluted in sterilized Milli-Q water $(30 \mu \mathrm{l} 50 \mathrm{mM}$ PUFAs and $120 \mu \mathrm{l}$ water) and overlaid on top of a seeded bacteria lawn in a $6 \mathrm{~cm}$ nematode growth media (NGM) plate. The plates were immediately dried in a dark laminar flow hood for 30 min. For fluorescence imaging, six hjSi3;hjSil12 or hjSi3;hjSil12 ok1126 L4 larvae were transferred onto each PUFAs- supplemented plate and allowed to lay eggs for 2 days. Two replicates were performed for each strain in each PUFAs-supplemented condition. For each replicate, 6-8 of their progenies at the L4 larval stage were imaged.

Generation of stable mammalian cell lines. COS7 cells stably expressing SEIP-1:: Venus or SEIP-1::V5-APEX2 were generated by the Sleeping Beauty (SB) transposon system ${ }^{46,47}$. Cells were co-transfected with pCMV(CAT)T7-SB100 that bore the SB Transposase and a plasmid based on pSBbi-Hyg (SEIP-1::Venus) or pSBbiRH (SEIP-1::V5-APEX2) using Lipofectamine 2000 (Life Technologies). Selection of stable integrants was facilitated by $0.5 \mathrm{mg} / \mathrm{mL}$ hygromycin for at least 7 days. The resulting cell population was further sorted using a Becton Dickinson Influx system and arbitrarily sub-divided into "Low", "Medium", and "High" fractions based on the fluorescence intensity of Venus (for SEIP-1::Venus cells) or TdTomato (for SEIP-1::V5- APEX2 cells). The "Low" fractions of all cell lines were used. For COS7 cells that constitutively co-expressed SEIP-1::Venus and mRuby::LiveDrop (constructed according to Wilfling et al. ${ }^{11}$ ) or PEX30::mRuby, LiveDrop or PEX30 were subcloned into pSBbi-BP vector. Each plasmid was co-transfected into cells that stably expressed SEIP-1::Venus as established above. Selection of stable integrants was facilitated by $10 \mu \mathrm{g} / \mathrm{mL}$ puromycin (InvivoGen) and $0.5 \mathrm{mg} / \mathrm{mL}$ hygromycin for at least 7 days. To constitutively express SEIP-1::Venus and inducibly express of SAR1a (H79G)::mRuby, COS7 cells were treated by procedures elaborated as above, but with a mouse sarla $(H 79 G):: m R u b y$ fusion sequence subcloned into the pSBtet-BP backbone.

Transmission electron microscopy. Chemical staining and dehydration of COS7 cells that stably expressed SEIP-1::V5-APEX2 were performed based on published methods ${ }^{47,79}$. Prior to fixation, cells were incubated with $400 \mu \mathrm{M}$ oleic acid-BSA complex (Sigma) for $24 \mathrm{~h}$. Fixation was conducted using $2.5 \%$ glutaraldehyde (Electron Microscopy Science) in wash buffer (100 mM sodium cacodylate with 2 $\mathrm{mM} \mathrm{CaCl}_{2}, \mathrm{pH} \mathrm{7.4)}$ ). For diaminobenzidine (DAB) staining, $5 \mathrm{mg} / \mathrm{mL}$ DAB free base (Sigma) stock solution dissolved in $0.1 \mathrm{M} \mathrm{HCl}$ was freshly diluted with $10 \mathrm{mM}$ $\mathrm{H}_{2} \mathrm{O}_{2}$ in cold wash buffer, and cells were incubated in the solution for $10 \mathrm{~min}$. SEIP-1::Venus stable cells were used as the negative control for assessing nonspecific DAB deposition. Two percent of osmium tetroxide (Electron Microscopy Science) staining was performed for $40 \mathrm{~min}$ in cold wash buffer, followed by incubation with chilled $2 \%$ uranyl acetate in $\mathrm{dd}_{2} \mathrm{O}$ overnight at $4{ }^{\circ} \mathrm{C}$. Infiltration was performed at room temperature with a progressive series $(10,25,50,75$, and $100 \%$ ) of EMBED-812 resin to ethanol dilutions. Specimens were incubated for at least $3 \mathrm{~h}$ in each dilution. After three changes of $100 \%$ Epon resin, samples were then transferred to a plastic mold and cured at $60^{\circ} \mathrm{C}$ for $40 \mathrm{~h}^{80}$. Cured blocks were then trimmed and sectioned with a diamond knife. In total, 150-nm thin sections were cut and mounted on copper slot grids coated with formvar and further imaged with a Hitachi H-7650 transmission electron microscope operated at $80 \mathrm{kV}$.

For 3D model reconstruction, serial images were stacked and aligned using IMOD software package (Boulder Laboratory of 3D Electron Microscopy of the Cell, University of Colorado at Boulder). 3D modeling of ER without obvious DAB/APEX2 staining and LDs were performed using 3dmod graphic module while DAB/APEX2 precipitates were reconstructed using the auto-contour function under high contrast with a threshold level.

Immunostaining endogenous SEIP-1 in dissected C. elegans. Animals were dissected, fixed and immunostained according to published methods with minor modifications. Briefly, 200 L4 larval animals of each genotype were dissected in $1 \times$ PBS/0.0005\% Triton X-100 (Sigma) with $0.2 \mathrm{mM}$ levamisole for intestine extrusion and fixed for $4 \mathrm{~h}$ at $4{ }^{\circ} \mathrm{C}$ in $2 \%$ paraformaldehyde. At room temperature, the fixed animals were permeabilized for $1 \mathrm{~h}$ in $1 \times \mathrm{PBS} / 0.1 \%$ Triton X-100, followed by a $1 \mathrm{~h}$ blocking in $1 \times \mathrm{PBS} / 0.1 \%$ Tween $20 / 1 \%$ bovine serum albumin (BSA). Primary (1:100 diluted monoclonal anti-FLAG antibody (M2) (Sigma)) and secondary antibody (1:100 diluted Alexa Fluor 488 Donkey anti-Mouse IgG $(\mathrm{H}+\mathrm{L})$ (Invitrogen)) incubations were both performed overnight at $4{ }^{\circ} \mathrm{C}$ in $1 \times \mathrm{PBS} / 0.1 \%$ Tween $20 / 1 \%$ BSA. Sample was post-stained by HCS LipidTOX ${ }^{\mathrm{TM}}$ red neutral lipid stain (Invitrogen) and Hoechst 33342 (Invitrogen) according to manufacturer's instructions and mounted onto a 35-mm MatTek glass-bottom dish coated with poly-D-lysine prior to imaging.

Total lipid extraction. Sample was prepared and extracted according to published methods with slight modifications ${ }^{41}$. For total lipid extraction from bacteria, $\sim 15 \mathrm{~mL}$ overnight culture was concentrated $10 \times$ and spread on a $10 \mathrm{~cm}$ NGM plate. The plate was immediately dried in a laminar flow hood and incubated at $20^{\circ} \mathrm{C}$ for 2 days. Bacteria was rinsed off the plate, pelleted and washed three times using $0.9 \%$ $\mathrm{NaCl}$ solution. Two plates were combined as one sample, at least three samples were analyzed for each bacterial strain. After the last wash, the pellet was resuspended and sonicated in ice water bath for five times at $40 \%$ amplitude with a $2 \mathrm{~min}$ on $/ 30 \mathrm{~s}$ off cycle. One-tenth of the lysates were sampled to determine the soluble protein concentration using the BCA Protein Assay Kit (Pierce) and used for subsequent data normalization. Known amount of palmitic acid-D31 (Sigma) was added to the remaining lysates in order to control for extraction efficiency, sample loss and subsequent trans-methylation efficiency. One volume of the remaining lysates were subsequently extracted using ten volumes of pre-chilled Folch solution (chloroform: methanol, 2:1, v-v) followed by five volumes of chloroform. Total lipids were pooled 
together from bottom chloroform phases into a clean glass vial with a PTFE cap and dried under a gentle stream of nitrogen at $37^{\circ} \mathrm{C}$.

For FAs analysis of C. elegans, $\sim 4000$ newly hatched L1 larvae were seeded onto each plate prepared as described above and allowed to grow to the late-L4 stage. L4 larvae were harvested and washed four times with $1 \times \mathrm{PBS} / 0.001 \%$ Tween 20 . Worm pellets were snap- frozen and stored in $-80^{\circ} \mathrm{C}$ prior to lipid extraction. Each plate of worms was used as one sample, at least four independent samples were used for each condition. For lipid extraction, $500 \mu \mathrm{l}$ of 1 xPBS/0.001\% Tween $20 / 0.001 \%$ butylated hydroxytoluene (BHT) was added to each sample pellet and sonicated in ice water bath for 15 times at $40 \%$ amplitude with a $2 \mathrm{~min}$ on $/ 30 \mathrm{sec}$ off cycle. One hundred microliters of the sonicated sample was mixed with an equal volume of $4 \times$ SDS buffer (8\% SDS, $200 \mathrm{mM}$ Tris-HCl pH 6.8) and denatured at $95^{\circ} \mathrm{C}$ for $10 \mathrm{~min}$. Concentration of soluble protein was determined using the BCA Protein Assay Kit (Pierce) and used for subsequent data normalization. Known amount of heptadecanoic acid (C17:0, Nu-Chek Prep) was added to the remaining lysates in order to control for extraction efficiency, sample loss and subsequent transmethylation efficiency. The remaining $400 \mu \mathrm{l}$ worm lysate was subsequently subjected to total lipid extraction as described for bacteria above.

Preparation of fatty acid methyl esters (FAMEs). Dried lipids were re-dissolved in $1.95 \mathrm{~mL}$ methanol. Trans-methylation was carried out by adding $50 \mu \mathrm{L}$ sulfuric acid and incubating the sample mixture at $80^{\circ} \mathrm{C}$ for $1 \mathrm{~h}$. After adding $1.5 \mathrm{~mL}$ Milli$\mathrm{Q}$ water, fatty acid methyl esters (FAMEs) were extracted with 200 or $400 \mu \mathrm{L}$ $\mathrm{n}$-hexane (RCI Labscan) and transferred into autosampler vials with inserts.

GC-MS analysis. GC-MS analysis was performed on an Agilent 7890B gas chromatograph equipped with an Agilent 5977 A mass spectrometer, an Agilent 7693 autosampler, and an Agilent DB-23 $30 \mathrm{~m} \times 0.25 \mathrm{~mm} \times 0.25 \mu \mathrm{m}$ GC column (\#1222332; Agilent). One microliter of sample was injected in splitless mode. Carrier gas helium was set at a constant flow rate of $1.6 \mathrm{~mL} / \mathrm{min}$. The inlet temperature was $220^{\circ} \mathrm{C}$. The column temperature was maintained at $100^{\circ} \mathrm{C}$ for $1 \mathrm{~min}$, and then increased to $180^{\circ} \mathrm{C}$ at $10^{\circ} \mathrm{C} / \mathrm{min}$, then held at $180^{\circ} \mathrm{C}$ for $5 \mathrm{~min}$. Finally, temperature was raised to $220^{\circ} \mathrm{C}$ at $5{ }^{\circ} \mathrm{C} / \mathrm{min}$, and held at $220^{\circ} \mathrm{C}$ for $3 \mathrm{~min}$. The retention time of each FAMEs were identified when compared with the peaks of FAME standards that purchased from Nu-Chek Prep or by using Supelco 37 Component FAME Mix. Mass spectra of unidentified peaks were matched against NIST14 library. The response factors (RFs) for each FAMEs relative to the internal standard were determined from the constructed calibration curve. Quantitation was achieved by relating the peak area of each FAME with the spiked internal standard.

Reporting summary. Further information on research design is available in the Nature Research Reporting Summary linked to this article.

\section{Data availability}

The data that support the findings of this study are available from the corresponding author upon request.

Received: 25 July 2018 Accepted: 3 June 2019

Published online: 01 July 2019

\section{References}

1. Fujimoto, T. \& Parton, R. G. Not just fat: the structure and function of the lipid droplet. Cold Spring Harb. Perspect. Biol. 3, a004838 (2011).

2. Mak, H. Y. Lipid droplets as fat storage organelles in Caenorhabditis elegans: thematic review series: lipid droplet synthesis and metabolism: from yeast to man. J. Lipid Res. 53, 28-33 (2012).

3. Walther, T. C. \& Farese, R. V. Jr. Lipid droplets and cellular lipid metabolism. Annu. Rev. Biochem. 81, 687-714 (2012).

4. Krahmer, N. et al. Phosphatidylcholine synthesis for lipid droplet expansion is mediated by localized activation of CTP: phosphocholine cytidylyltransferase. Cell Metab. 14, 504-515 (2011).

5. Tauchi-Sato, K., Ozeki, S., Houjou, T., Taguchi, R. \& Fujimoto, T. The surface of lipid droplets is a phospholipid monolayer with a unique fatty acid composition. J. Biol. Chem. 277, 44507-44512 (2002).

6. Yang, L. et al. The proteomics of lipid droplets: structure, dynamics, and functions of the organelle conserved from bacteria to humans. J. Lipid Res. 53, 1245-1253 (2012).

7. Cermelli, S., Guo, Y., Gross, S. P. \& Welte, M. A. The lipid-droplet proteome reveals that droplets are a protein-storage depot. Curr. Biol. CB 16, 1783-1795 (2006).

8. O'Byrne, S. M. \& Blaner, W. S. Retinol and retinyl esters: biochemistry and physiology. J. Lipid Res. 54, 1731-1743 (2013).

9. Thiam, A. R. \& Beller, M. The why, when and how of lipid droplet diversity. J. Cell Sci. 130, 315-324 (2017).
10. Thul, P. J. et al. Targeting of the Drosophila protein CG2254/Ldsdh1 to a subset of lipid droplets. J. Cell Sci. 130, 3141-3157 (2017).

11. Wilfling, F. et al. Triacylglycerol synthesis enzymes mediate lipid droplet growth by relocalizing from the ER to lipid droplets. Dev. Cell 24, 384-399 (2013).

12. Robenek, H. et al. Compartmentalization of proteins in lipid droplet biogenesis. Biochim. Biophys. Acta 1791, 408-418 (2009).

13. Blanchette-Mackie, E. J. et al. Perilipin is located on the surface layer of intracellular lipid droplets in adipocytes. J. Lipid Res. 36, 1211-1226 (1995).

14. $\mathrm{Xu}, \mathrm{N}$. et al. The FATP1-DGAT2 complex facilitates lipid droplet expansion at the ER-lipid droplet interface. J. Cell Biol. 198, 895-911 (2012).

15. Jacquier, N. et al. Lipid droplets are functionally connected to the endoplasmic reticulum in Saccharomyces cerevisiae. J. Cell Sci. 124, 2424-2437 (2011).

16. Datta, S., Liu, Y., Hariri, H., Bowerman, J. \& Henne, W. M. Cerebellar ataxia disease-associated Snx14 promotes lipid droplet growth at ER-droplet contacts. J. Cell Biol. https://doi.org/10.1083/jcb.201808133 (2019).

17. $\mathrm{Xu}, \mathrm{D}$. et al. Rab18 promotes lipid droplet (LD) growth by tethering the ER to LDs through SNARE and NRZ interactions. J. Cell Biol. 217, 975-995 (2018).

18. Coleman, R. A. \& Lee, D. P. Enzymes of triacylglycerol synthesis and their regulation. Prog. Lipid Res. 43, 134-176 (2004).

19. Kuerschner, L., Moessinger, C. \& Thiele, C. Imaging of lipid biosynthesis: how a neutral lipid enters lipid droplets. Traffic Cph. Den. 9, 338-352 (2008).

20. Klemm, R. W. et al. A conserved role for atlastin GTPases in regulating lipid droplet size. Cell Rep. 3, 1465-1475 (2013).

21. Magré, J. et al. Identification of the gene altered in Berardinelli-Seip congenital lipodystrophy on chromosome 11q13. Nat. Genet. 28, 365-370 (2001).

22. Cartwright, B. R. \& Goodman, J. M. Seipin: from human disease to molecular mechanism. J. Lipid Res. 53, 1042-1055 (2012).

23. Chen, W. et al. Berardinelli-Seip congenital lipodystrophy $2 /$ seipin is a cellautonomous regulator of lipolysis essential for adipocyte differentiation. Mol. Cell. Biol. 32, 1099-1111 (2012).

24. Payne, V. A. et al. The human lipodystrophy gene BSCL2/seipin may be essential for normal adipocyte differentiation. Diabetes 57, 2055-2060 (2008).

25. Yang, W. et al. Seipin differentially regulates lipogenesis and adipogenesis through a conserved core sequence and an evolutionarily acquired Cterminus. Biochem. J. https://doi.org/10.1042/BJ20121870 (2013).

26. Binns, D., Lee, S., Hilton, C. L., Jiang, Q.-X. \& Goodman, J. M. Seipin is a discrete homooligomer. Biochemistry 49, 10747-10755 (2010).

27. Sui, X. et al. Cryo-electron microscopy structure of the lipid droplet-formation protein seipin. J. Cell Biol. https://doi.org/10.1083/jcb.201809067(2018).

28. Yan, R. et al. Human SEIPIN binds anionic phospholipids. Dev. Cell $\mathbf{4 7}$ 248-256.e4 (2018)

29. Cai, Y. et al. Arabidopsis SEIPIN proteins modulate triacylglycerol accumulation and influence lipid droplet proliferation. Plant Cell 27, 2616-2636 (2015)

30. Fei, W. et al. Fld1p, a functional homologue of human seipin, regulates the size of lipid droplets in yeast. J. Cell Biol. 180, 473-482 (2008).

31. Salo, V. T. et al. Seipin regulates ER-lipid droplet contacts and cargo delivery. EMBO J. 35, 2699-2716 (2016)

32. Szymanski, K. M. et al. The lipodystrophy protein seipin is found at endoplasmic reticulum lipid droplet junctions and is important for droplet morphology. Proc. Natl Acad. Sci. USA 104, 20890-20895 (2007).

33. Fei, W. et al. A role for phosphatidic acid in the formation of 'supersized' lipid droplets. PLoS Genet. 7, e1002201 (2011).

34. Wang, H. et al. Seipin is required for converting nascent to mature lipid droplets. eLife 5, e16582 (2016).

35. van Meer, G., Voelker, D. R. \& Feigenson, G. W. Membrane lipids: where they are and how they behave. Nat. Rev. Mol. Cell Biol. 9, 112-124 (2008).

36. Antonny, B., Vanni, S., Shindou, H. \& Ferreira, T. From zero to six double bonds: phospholipid unsaturation and organelle function. Trends Cell Biol. 25, 427-436 (2015).

37. Sledzinski, T. et al. Identification of cyclopropaneoctanoic acid 2-hexyl in human adipose tissue and serum. Lipids 48, 839-848 (2013).

38. Tsirigos, K. D., Peters, C., Shu, N., Käll, L. \& Elofsson, A. The TOPCONS web server for consensus prediction of membrane protein topology and signal peptides. Nucleic Acids Res. 43, W401-W407 (2015).

39. Lundin, C. et al. Membrane topology of the human seipin protein. FEBS Lett. 580, 2281-2284 (2006).

40. Li, X. et al. Integrated femtosecond stimulated Raman scattering and twophoton fluorescence imaging of subcellular lipid and vesicular structures. J. Biomed. Opt. 20, 110501 (2015).

41. Zhang, S. O. et al. Genetic and dietary regulation of lipid droplet expansion in Caenorhabditis elegans. Proc. Natl Acad. Sci. USA 107, 4640-4645 (2010).

42. Lam, S. M., Wang, Z., Li, J., Huang, X. \& Shui, G. Sequestration of polyunsaturated fatty acids in membrane phospholipids of Caenorhabditis elegans dauer larva attenuates eicosanoid biosynthesis for prolonged survival. Redox Biol. 12, 967-977 (2017). 
43. $\mathrm{Na}, \mathrm{H}$. et al. Identification of lipid droplet structure-like/resident proteins in Caenorhabditis elegans. Biochim. Biophys. Acta 1853, 2481-2491 (2015).

44. Wolinski, H., Kolb, D., Hermann, S., Koning, R. I. \& Kohlwein, S. D. A role for seipin in lipid droplet dynamics and inheritance in yeast. J. Cell Sci. 124, 3894-3904 (2011).

45. Wang, S. et al. Seipin and the membrane-shaping protein Pex30 cooperate in organelle budding from the endoplasmic reticulum. Nat. Commun. 9, 2939 (2018).

46. Kowarz, E., Löscher, D. \& Marschalek, R. Optimized Sleeping Beauty transposons rapidly generate stable transgenic cell lines. Biotechnol. J. 10, 647-653 (2015).

47. Lam, S. S. et al. Directed evolution of APEX2 for electron microscopy and proximity labeling. Nat. Methods 12, 51-54 (2015).

48. Labrousse, A. M., Zappaterra, M. D., Rube, D. A. \& van der Bliek, A. M. C. elegans dynamin-related protein DRP-1 controls severing of the mitochondrial outer membrane. Mol. Cell 4, 815-826 (1999).

49. Ichishita, R. et al. An RNAi screen for mitochondrial proteins required to maintain the morphology of the organelle in Caenorhabditis elegans. J. Biochem. 143, 449-454 (2008).

50. Watts, J. L. \& Browse, J. Genetic dissection of polyunsaturated fatty acid synthesis in Caenorhabditis elegans. Proc. Natl Acad. Sci. USA 99, 5854-5859 (2002).

51. Grogan, D. W. \& Cronan, J. E. Cloning and manipulation of the Escherichia coli cyclopropane fatty acid synthase gene: physiological aspects of enzyme overproduction. J. Bacteriol. 158, 286-295 (1984).

52. Chang, Y. Y. \& Cronan, J. E. Membrane cyclopropane fatty acid content is a major factor in acid resistance of Escherichia coli. Mol. Microbiol. 33, 249-259 (1999).

53. Baba, T. et al. Construction of Escherichia coli K-12 in-frame, single-gene knockout mutants: the Keio collection. Mol. Syst. Biol. 2, 2006.0008 (2006).

54. Walker, A. K. et al. A conserved SREBP-1/phosphatidylcholine feedback circuit regulates lipogenesis in metazoans. Cell 147, 840-852 (2011).

55. Svensk, E. et al. PAQR-2 regulates fatty acid desaturation during cold adaptation in C. elegans. PLoS Genet. 9, e1003801 (2013).

56. Wang, Z. et al. Specific fluorescence probes for lipid droplets based on simple AIEgens. ACS Appl. Mater. Interfaces 8, 10193-10200 (2016).

57. Soni, K. G. et al. Coatomer-dependent protein delivery to lipid droplets. J. Cell Sci. 122, 1834-1841 (2009).

58. Lee, J. H. et al. Lipid droplet protein LID-1 mediates ATGL-1-dependent lipolysis during fasting in Caenorhabditis elegans. Mol. Cell. Biol. 34, 4165-4176 (2014).

59. Young, S. G. \& Zechner, R. Biochemistry and pathophysiology of intravascular and intracellular lipolysis. Genes Dev. 27, 459-484 (2013).

60. Pagac, M. et al. SEIPIN regulates lipid droplet expansion and adipocyte development by modulating the activity of glycerol-3-phosphate acyltransferase. Cell Rep. 17, 1546-1559 (2016).

61. Han, S., Binns, D. D., Chang, Y.-F. \& Goodman, J. M. Dissecting seipin function: the localized accumulation of phosphatidic acid at ER/LD junctions in the absence of seipin is suppressed by $\operatorname{Seil} \mathrm{p}(\Delta \mathrm{Nterm})$ only in combination with Ldb16p. BMC Cell Biol. 16, 29 (2015).

62. Wolinski, H. et al. Seipin is involved in the regulation of phosphatidic acid metabolism at a subdomain of the nuclear envelope in yeast. Biochim. Biophys. Acta 1851, 1450-1464 (2015).

63. Wang, C.-W., Miao, Y.-H. \& Chang, Y.-S. Control of lipid droplet size in budding yeast requires the collaboration between Fld1 and Ldb16. J. Cell Sci. 127, 1214-1228 (2014)

64. Grippa, A. et al. The seipin complex Fld1/Ldb16 stabilizes ER-lipid droplet contact sites. J. Cell Biol. 211, 829-844 (2015).

65. Teixeira, V. et al. Regulation of lipid droplets by metabolically controlled Ldo isoforms. J. Cell Biol. 217, 127-138 (2018).

66. Eisenberg-Bord, M. et al. Identification of seipin-linked factors that act as determinants of a lipid droplet subpopulation. J. Cell Biol. 217, 269-282 (2018).

67. Bersuker, K. et al. A proximity labeling strategy provides insights into the composition and dynamics of lipid droplet proteomes. Dev. Cell 44, 97-112.e7 (2018).

68. Castro, I. G. et al. Promethin is a conserved seipin partner protein. Cells 8, 268 (2019).

69. Pinot, M. et al. Lipid cell biology. Polyunsaturated phospholipids facilitate membrane deformation and fission by endocytic proteins. Science 345, 693-697 (2014).

70. Vanni, S., Hirose, H., Barelli, H., Antonny, B. \& Gautier, R. A sub-nanometre view of how membrane curvature and composition modulate lipid packing and protein recruitment. Nat. Commun. 5, 4916 (2014).

71. Joshi, A. S. et al. Lipid droplet and peroxisome biogenesis occur at the same ER subdomains. Nat. Commun. 9, 2940 (2018).

72. Sonnenburg, J. L. \& Bäckhed, F. Diet-microbiota interactions as moderators of human metabolism. Nature 535, 56-64 (2016).
73. Lin, C.-C. J. \& Wang, M. C. Microbial metabolites regulate host lipid metabolism through NR5A-Hedgehog signalling. Nat. Cell Biol. 19, 550-557 (2017).

74. Wang, E. \& Hunter, C. P. SID-1 functions in multiple roles to support parental RNAi in Caenorhabditis elegans. Genetics 207, 547-557 (2017)

75. Peng, G. et al. Oleate blocks palmitate-induced abnormal lipid distribution, endoplasmic reticulum expansion and stress, and insulin resistance in skeletal muscle. Endocrinology 152, 2206-2218 (2011).

76. Li, X., Jiang, M., Lam, J. W. Y., Tang, B. Z. \& Qu, J. Y. Mitochondrial imaging with combined fluorescence and stimulated Raman scattering microscopy using a probe of the aggregation-induced emission characteristic. J. Am. Chem. Soc. 139, 17022-17030 (2017).

77. Ramachandran, P. V., Mutlu, A. S. \& Wang, M. C. Label-free biomedical imaging of lipids by stimulated Raman scattering microscopy. Curr. Protoc. Mol. Biol. 109, 30.3.1-17 (2015).

78. Zhang, P. et al. Proteomic study and marker protein identification of Caenorhabditis elegans lipid droplets. Mol. Cell. Proteom. MCP 11, 317-328 (2012).

79. Hung, V. et al. Spatially resolved proteomic mapping in living cells with the engineered peroxidase APEX2. Nat. Protoc. 11, 456-475 (2016).

80. Kang, B.-H. Electron microscopy and high-pressure freezing of Arabidopsis. Methods Cell Biol. 96, 259-283 (2010).

\section{Acknowledgements}

We thank John T. Crowl and Daniel Capek for their contributions in the C. elegans genetic screen, Shohei Mitani for providing mutant strain, Lidan Zeng for transgenic strains, Sean McKinney, Yusong Guo, Meng Wang, Justin Law, and Jetty Lee for reagents and technical advice, the Molecular Biology core at the Stowers Institute for Medical Research for whole-genome sequencing, Tom Rapoport, Gunther Hollopeter, and David Banfield for comments. This work was supported by the Stowers Institute for Medical Research, and grants 662013, 16101614 and HKUST12/CRF/13G from the Hong Kong Research Grants Council to H.Y.M., grants U1402225 and 31571388 from the National Natural Science Foundation of China to P.L., and a grant CAS16SC01 from the Croucher Foundation to H.Y.M. and P.L.

\section{Author contributions}

Z.C. was responsible for Figs. 1 (a, b, d, e), 2 (b-g, i), 3 (a, b, d-g), 4 (b-g, i-l), 5, 6, 7, Supp. Figs. 1 (a, d-g), 2 (a-c, h, i), 3 (a-d), 4 (a, b, e, f, h-j), 5, 6 (a-g, j, k), 7, 8, and 9. Y. H. was responsible for Figs. 1c and 2a. C.W.F. was responsible for Figs. 1 (f, g), 2h, Supp. Figs. 1 (h-k), 2 (d-g), 4 (c, d, g), and 6 (h, i). Y.Y.L. was responsible for Figs. 4l, 5f, and Supp. Fig. 5b. P.W. and B.H.K. were responsible for Fig. 3 (d-g) and Supp. Fig. 3 (e-l). X. L. and J.Q. were responsible for developing the SRS system and Figs. $1 \mathrm{~b}$ and $2 \mathrm{~d}$. K.X. and P.L. were responsible for Fig. 2e. W.J.L. was responsible for Fig. 4 h and Supp. Fig. 2j. Y.Q. was responsible for Fig. 3c. G.S. was responsible for Supp. Fig. 1 (b, c). B.Z.T. provided LD dyes. Y.H. was responsible for the initial characterization of anti-SEIP-1 antibodies, all seip-1 transgenes, and preliminary experiments for Figs. 1, 2, 4, Supp. Figs 1, and 2. H. Y.M. and Y.H. conducted genetic screens, genetic mapping, and molecular cloning of $C$. elegans mutants. H.Y.M. wrote the paper with inputs from Z.C.

\section{Additional information}

Supplementary Information accompanies this paper at https://doi.org/10.1038/s41467 019-10835-4.

Competing interests: The authors declare no competing interests.

Reprints and permission information is available online at http://npg.nature.com/ reprintsandpermissions/

Publisher's note: Springer Nature remains neutral with regard to jurisdictional claims in published maps and institutional affiliations.

Open Access This article is licensed under a Creative Commons Attribution 4.0 International License, which permits use, sharing, adaptation, distribution and reproduction in any medium or format, as long as you give appropriate credit to the original author(s) and the source, provide a link to the Creative Commons license, and indicate if changes were made. The images or other third party material in this article are included in the article's Creative Commons license, unless indicated otherwise in a credit line to the material. If material is not included in the article's Creative Commons license and your intended use is not permitted by statutory regulation or exceeds the permitted use, you will need to obtain permission directly from the copyright holder. To view a copy of this license, visit http://creativecommons.org/ licenses/by/4.0/.

(C) The Author(s) 2019 